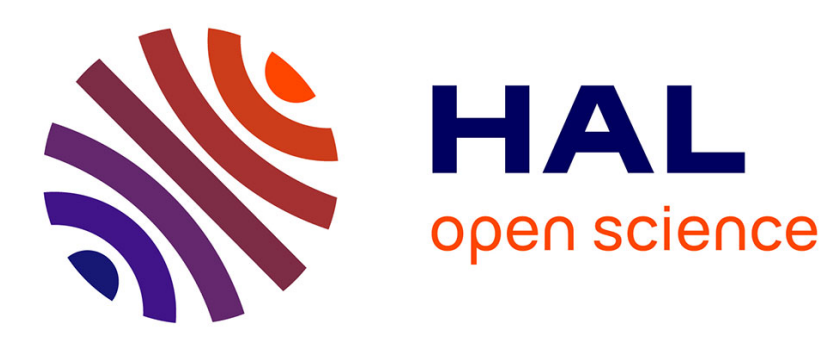

\title{
Explicit coupling schemes for a fluid-fluid interaction problem arising in hemodynamics
}

Miguel Angel Fernández, Jean-Frédéric Gerbeau, Saverio Smaldone

\section{To cite this version:}

Miguel Angel Fernández, Jean-Frédéric Gerbeau, Saverio Smaldone. Explicit coupling schemes for a fluid-fluid interaction problem arising in hemodynamics. SIAM Journal on Scientific Computing, 2014, 36 (6), pp.2557-2583. 10.1137/130948653 . hal-00915213v2

\section{HAL Id: hal-00915213 \\ https://hal.inria.fr/hal-00915213v2}

Submitted on 1 May 2014

HAL is a multi-disciplinary open access archive for the deposit and dissemination of scientific research documents, whether they are published or not. The documents may come from teaching and research institutions in France or abroad, or from public or private research centers.
L'archive ouverte pluridisciplinaire HAL, est destinée au dépôt et à la diffusion de documents scientifiques de niveau recherche, publiés ou non, émanant des établissements d'enseignement et de recherche français ou étrangers, des laboratoires publics ou privés. 
Explicit coupling schemes for a fluid-fluid interaction problem arising in hemodynamics

Miguel A. Fernández, Jean-Frédéric Gerbeau, Saverio Smaldone

\section{RESEARCH}

REPORT

$N^{\circ} 8415$

December 2013

Project-Team Reo 



\title{
Givita
}

\section{Explicit coupling schemes for a fluid-fluid interaction problem arising in hemodynamics}

\author{
Miguel A. Fernández ${ }^{* \dagger}$, Jean-Frédéric Gerbeau*†, Saverio \\ Smaldone*† \\ Project-Team Reo \\ Research Report $\mathrm{n}^{\circ} 8415$ - December 2013 - 31 pages
}

\begin{abstract}
In this work we propose a new approach to the loosely coupled time-marching of a fluid-fluid interaction problems involving the incompressible Navier-Stokes equations. The methods combine a specific explicit Robin-Robin treatment of the interface coupling with a weakly consistent interface pressure stabilization in time. A priori energy estimates guaranteeing stability of the splitting are obtained for a total pressure formulation of the coupled problem. The performance of the proposed schemes is illustrated on several numerical experiments related to simulation of aortic blood flow.
\end{abstract}

Key-words: fluid-structure interaction, incompressible fluid, time-discretization, loosely coupled scheme, Robin-Robin scheme, finite element method, Nitsche's method.

Work partially supported by the French National Research Agency (ANR) through the EXIFSI project (ANR-12-JS01-0004). The authors thank the anonymous reviewers for their valuable comments and suggestions.

* Inria, REO project-team, Rocquencourt - B.P. 105, F-78153 Le Chesnay cedex, France

† UPMC Université Paris VI, REO project-team, UMR 7958 LJLL, F-75005 Paris, France

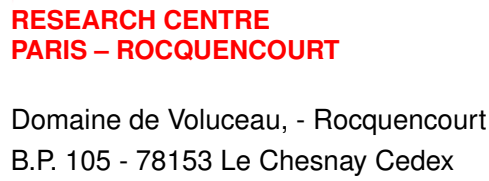




\section{Schémas de couplage explicites pour un problème d'interaction fluide-fluide en hémodynamique}

Résumé : Dans cet article, nous proposons une nouvelle approche pour le couplage faible de deux fluides incompressibles régis par les équations de Navier-Stokes. Ces méthodes combinent un traitement spécifique Robin-Robin explicite des conditions d'interface avec une stabilisation de la pression à l'interface faiblement consistante en temps. Une estimation d'énergie a priori garantissant la stabilité du dćouplage en temps est obtenue pour une formulation en pression totale du système couplé. Les propriétés des méthodes proposées sont illustrées dans des expériences numériques motivées par la simulation de la circulation du sang dans la aorte.

Mots-clés : interaction fluide-fluide, fluide incompressible, discrétisation en temps, schéma de couplage faible, schéma Robin-Robin, méthode des éléments finis, méthode de Nitsche. 


\section{Introduction}

Global and local features in hemodynamics are generally described by coupling dimensionally heterogeneous models of blood flow. In this geometrical multiscale paradigm, the three-dimensional (3D) Navier-Stokes equations are usually coupled with one-dimensional (1D) and/or zerodimensional (0D) models that account for the parts before and after the 3D compartment (see, e.g., $[6,16,32])$. In this work, we are interested in coupling 3D compartments. Indeed, in some of them, it may be sufficient to consider a standard Navier-Stokes solver. But it is sometimes necessary to develop ad-hoc software to handle complex configurations. For instance, if a detailed modeling of blood flow in the left ventricle is sought for, the 3D model must incorporate additional features, such as the deformation of the cardiac cavity and the opening-closing dynamics of the aortic valves, which requires a very specific solver. Another example is provided by the simulation of endo-vascular devices (e.g., stents, coils) or vascular pathologies (e.g., aortic dissection, atherosclerotic plaques development, vascular wall remodelling) that may also require the development of specific software. In this context, it may be of practical interest to couple different $3 \mathrm{D}$ codes. The motivation of this work is therefore the partitioning of the 3D fluid computational domain into several complementary non-overlapping subdomains, in which these additional modeling features may take place. As an alternative to standard domain decomposition approaches, we propose to advance in time the system via specific explicit coupling (or loosely coupled) strategies that allow the uncoupled time-stepping of the sub-compartments.

As a preliminary step in this direction, in this work we focus on the case of two sub-compartments described by the incompressible Navier-Stokes equations. The two sub-problems are coupled, across their shared (artificial) interface, by standard kinematic/kinetic conditions, viz., continuity of velocity and stress. We address the uncoupled time-marching of the two subcompartments via explicit coupling schemes. Note that this approach clearly differs from [26, 5], where partitioned iterative procedures acting on averaged/integrated interface quantities are proposed.

To this purpose, a major difficulty that has to be faced is related to the artificial energy transfers (unbalance of static and/or dynamic pressure power) induced by the interface timesplitting, which can lead to numerical instabilities. For instance, if a standard Dirichlet-Neumann scheme is foreseen (see [18]), the splitting introduces an unbalance of static powers across the interface. Relaxation of the kinematic constraint improves the situation, but with the payoff of an uncontrolled dynamic pressure power across the interface. This issue does not appear in fluidfluid interaction models based on rigid-lid conditions, commonly used in oceanography modeling (see, e.g., $[7,13,14,30]$ ), for which the normal velocity component vanishes on the interface.

In this work, the unbalanced static pressure power is controlled via a specific explicit RobinRobin treatment of the interface coupling (based on interface Nitsche's method) and a weakly consistent interface pressure stabilization in time. Basically, this approach extends the loosely coupled schemes introduced in [8, 10] for FSI problems to fluid-fluid interaction problems. We also show that the second source of instabilities (unbalanced dynamic pressure power) can be treated, in a natural way, by applying this method to a total pressure formulation of the NavierStokes equations.

Several numerical experiments, related to the simulation of aortic blood flow, illustrate the accuracy and performance of the schemes proposed in two non-overlapping sub-domains. An example in which the computational domain is partitioned in three sub-domains is reported for one of the staggered methods.

The paper is organized as follows. In the next section, the incompressible Navier-Stokes equations are split into two sub-problems coupled through an artificial interface. A Nitsche's space semi-discrete formulation of this fluid-fluid interaction problem is presented in Section 3. 
Section 4 is devoted to the time-dicretization. Different implicit and explicit coupling strategies are presented, in the static and total pressure formulations, and their corresponding discrete energy balances is derived. The numerical experiments are reported in Section 5. Section 6 summarizes the conclusions.

\section{Coupled fluid-fluid problem}

Let $\Omega \subset \mathbb{R}^{d}, d=2,3$ be a bounded fixed domain and a fluid modelled by the incompressible Navier-Stokes equations. The velocity $\mathbf{u}: \Omega \times \mathbb{R}^{+} \rightarrow \mathbb{R}^{d}$ and the pressure $p: \Omega \times \mathbb{R}^{+} \rightarrow \mathbb{R}$ satisfy:

$$
\begin{aligned}
& \rho \partial_{t} \mathbf{u}+\rho \mathbf{u} \cdot \nabla \mathbf{u}-\nabla \cdot \boldsymbol{\sigma}(\mathbf{u}, p)=0, \quad \text { in } \Omega, \\
& \nabla \cdot \mathbf{u}=0, \quad \text { in } \Omega, \\
& \mathbf{u}=\mathbf{0}, \quad \text { on } \partial \Omega,
\end{aligned}
$$

with the initial conditions $\mathbf{u}(0)=\mathbf{u}_{0}$. The term $\boldsymbol{\sigma}(\mathbf{u}, p)=-p \mathbf{I}+2 \mu \boldsymbol{\epsilon}(\mathbf{u})$, where $\boldsymbol{\epsilon}(\mathbf{u})=(\nabla \mathbf{u}+$ $\left.(\nabla \mathbf{u})^{\mathrm{T}}\right) / 2$, is the fluid stress tensor, and $\rho$ and $\mu$ stand for the fluid density and the dynamic viscosity respectively. For the sake of the analysis, homogeneous Dirichlet conditions are enforced on the whole boundary $\partial \Omega$. More realistic boundary conditions are considered in the numerical experiments of Section 5 .

Problem (1) can be partitioned into two sub-problems defined in two non-overlapping subdomains, $\Omega_{1} \subset \mathbb{R}^{d}$ and $\Omega_{2} \subset \mathbb{R}^{d}$, with $\bar{\Omega}=\bar{\Omega}_{1} \cup \bar{\Omega}_{2}$ and $\Omega_{1} \cap \Omega_{2}=\emptyset$. The two sub-domains are separated by an interface $\Sigma:=\partial \Omega_{1} \cap \partial \Omega_{2}$, and $\Gamma_{1}:=\partial \Omega_{1} \backslash \Sigma, \Gamma_{2}:=\partial \Omega_{2} \backslash \Sigma$.

The new formulation of the problem reads: find the velocity $\mathbf{u}_{1}: \Omega_{1} \times \mathbb{R}^{+} \rightarrow \mathbb{R}^{d}$ and the pressure $p_{1}: \Omega_{1} \times \mathbb{R}^{+} \rightarrow \mathbb{R}$ such that

$$
\begin{aligned}
\rho \partial_{t} \mathbf{u}_{1}+\rho \mathbf{u}_{1} \cdot \nabla \mathbf{u}_{1}-\nabla \cdot \boldsymbol{\sigma}\left(\mathbf{u}_{1}, p_{1}\right) & =0, & & \text { in } \Omega_{1}, \\
\nabla \cdot \mathbf{u}_{1} & =0, & & \text { in } \Omega_{1}, \\
\mathbf{u}_{1} & =\mathbf{0}, & & \text { on } \Gamma_{1},
\end{aligned}
$$

find the velocity $\mathbf{u}_{2}: \Omega_{2} \times \mathbb{R}^{+} \rightarrow \mathbb{R}^{d}$ and the pressure $p_{2}: \Omega_{2} \times \mathbb{R}^{+} \rightarrow \mathbb{R}$, such that

$$
\begin{aligned}
& \rho \partial_{t} \mathbf{u}_{2}+\rho \mathbf{u}_{2} \cdot \nabla \mathbf{u}_{2}-\nabla \cdot \boldsymbol{\sigma}\left(\mathbf{u}_{2}, p_{2}\right)=0, \text { in } \Omega_{2}, \\
& \nabla \cdot \mathbf{u}_{2}=0, \quad \text { in } \Omega_{2}, \\
& \mathbf{u}_{2}=\mathbf{0}, \quad \text { on } \Gamma_{2} \text {. }
\end{aligned}
$$

The solutions in the two sub-domains are coupled through the usual kinematic and kinetic conditions:

$$
\left\{\begin{aligned}
\mathbf{u}_{1}=\mathbf{u}_{2}, & \text { on } \quad \Sigma \\
\boldsymbol{\sigma}\left(\mathbf{u}_{2}, p_{2}\right) \mathbf{n}_{2}=-\boldsymbol{\sigma}\left(\mathbf{u}_{1}, p_{1}\right) \mathbf{n}_{1}, & \text { on } \quad \Sigma
\end{aligned}\right.
$$

with $\mathbf{n}_{1}, \mathbf{n}_{2}$ the outward-pointing unit normal vectors on $\partial \Omega_{1}$ and $\partial \Omega_{2}$ respectively.

\section{Space semi-discretization via interface Nitsche's method}

The time splitting schemes introduced in the following sections are derived from a space semidiscrete formulation of (2)-(4) based on interface Nitsche's method (see, e.g., [20, 2]). In other words, conforming finite element approximations are used in each sub-system, which do not match at the interface, and the interface coupling (4) is enforced in a consistent fashion $\grave{a}$ la Nitsche. A 
salient feature of this approach, compared to a standard conforming finite element approximation, is that the artificial interface power, generated by the time splitting of the interface coupling, can be controlled directly by the consistent numerical dissipation of interface Nitsche's method (see $[8,10])$.

Nitsche's method, originally proposed to weakly enforce Dirichlet boundary conditions in elliptic problems (see [29]), has been extended to a number of interface problems arising in computational mechanics (see, e.g., [20] for a review), including domain decomposition [2], StokesDarcy coupling [11], elliptic-hyperbolic problems [12], fluid-structure interaction [21, 22, 8, 10] and, more recently, interface problems with unfitted meshes (see, e.g., [19, 27, 9]).

\subsection{Monolithic formulation}

Let be $\left\{\mathcal{T}_{1, h}\right\}_{h>0}$ and $\left\{\mathcal{T}_{2, h}\right\}_{h>0}$ two families of regular finite element triangulation for $\Omega_{1}$ and $\Omega_{2}$ respectively, with typical diameter $h$. Let $W_{i, h} \times Q_{i, h}$ denote an inf-sup stable, conforming finite element approximations of $\left[H^{1}\left(\Omega_{i}\right)\right]^{d} \times L^{2}\left(\Omega_{i}\right)$ for $i=1,2$. Let $V_{1, h}=W_{1, h} \cap\left[H_{\Gamma_{1}}^{1}\left(\Omega_{1}\right)\right]^{d}$ and $V_{2, h}=W_{2, h} \cap\left[H_{\Gamma_{2}}^{1}\left(\Omega_{2}\right)\right]^{d}$, where $\left[H_{\gamma}^{1}\left(\Omega_{i}\right)\right]^{d}$ denotes the space of $\left[H^{1}\left(\Omega_{i}\right)\right]^{d}$ functions vanishing on a part $\gamma$ of $\partial \Omega_{i}$.

The considered space semi-discrete formulation of problem (2)-(4) reads: for all $t>0$, find $\left(\mathbf{u}_{1, h}, p_{1, h}, \mathbf{u}_{2, h}, p_{2, h}\right) \in W_{1, h} \times Q_{1, h} \times W_{2, h} \times Q_{2, h}$ satisfying the essential boundary conditions and such that

$$
\begin{aligned}
& A_{1}\left[\mathbf{u}_{1, h} ;\left(\mathbf{u}_{1, h}, p_{1, h}\right),\left(\mathbf{v}_{1, h}, q_{1, h}\right)\right]+A_{2}\left[\mathbf{u}_{2, h} ;\left(\mathbf{u}_{2, h}, p_{2, h}\right),\left(\mathbf{v}_{2, h}, q_{2, h}\right)\right] \\
& \quad+C_{1}\left[\mathbf{u}_{1, h} ;\left(\mathbf{u}_{1, h}, \mathbf{u}_{2, h}\right), \mathbf{v}_{1, h}\right]+C_{2}\left[\left(\mathbf{u}_{2, h}, \mathbf{u}_{1, h}\right) ;\left(\mathbf{u}_{2, h}, \mathbf{u}_{1, h}\right), \mathbf{v}_{2, h}\right] \\
& \quad-\int_{\Sigma} \boldsymbol{\sigma}\left(\mathbf{u}_{2, h}, p_{2, h}\right) \mathbf{n}_{2} \cdot\left(\mathbf{v}_{2, h}-\mathbf{v}_{1, h}\right)+\frac{\gamma \mu}{h} \int_{\Sigma}\left(\mathbf{u}_{2, h}-\mathbf{u}_{1, h}\right) \cdot\left(\mathbf{v}_{2, h}-\mathbf{v}_{1, h}\right) \\
& \quad-\int_{\Sigma}\left(\mathbf{u}_{2, h}-\mathbf{u}_{1, h}\right) \cdot \mathbf{n}_{2} q_{2, h}=0,
\end{aligned}
$$

for all $\left(\mathbf{v}_{1, h}, q_{1, h}, \mathbf{v}_{2, h}, q_{2, h}\right) \in V_{1, h} \times Q_{1, h} \times V_{2, h} \times Q_{2, h}$, where

$$
\begin{aligned}
A_{1}\left[\mathbf{u}_{1, h} ;\left(\mathbf{u}_{1, h}, p_{1, h}\right),\left(\mathbf{v}_{1, h}, q_{1, h}\right)\right]:=\quad & \rho \int_{\Omega_{1}} \partial_{t} \mathbf{u}_{1, h} \cdot \mathbf{v}_{1, h}+\int_{\Omega_{1}} 2 \mu \boldsymbol{\epsilon}\left(\mathbf{u}_{1, h}\right): \boldsymbol{\epsilon}\left(\mathbf{v}_{1, h}\right) \\
& -\int_{\Omega_{1}} p_{1, h} \nabla \cdot \mathbf{v}_{1, h}+\int_{\Omega_{1}} q_{1, h} \nabla \cdot \mathbf{u}_{1, h} \\
& +\rho \int_{\Omega_{1}}\left(\mathbf{u}_{1, h} \cdot \nabla \mathbf{u}_{1, h}\right) \cdot \mathbf{v}_{1, h}, \\
& \rho \int_{\Omega_{2}} \partial_{t} \mathbf{u}_{2, h} \cdot \mathbf{v}_{2, h}+\int_{\Omega_{2}} 2 \mu \boldsymbol{\epsilon}\left(\mathbf{u}_{2, h}\right): \boldsymbol{\epsilon}\left(\mathbf{v}_{2, h}\right) \\
A_{2}\left[\mathbf{u}_{2, h} ;\left(\mathbf{u}_{2, h}, p_{2, h}\right),\left(\mathbf{v}_{2, h}, q_{2, h}\right)\right]:=\quad & \int_{\Omega_{2}} p_{2, h} \nabla \cdot \mathbf{v}_{2, h}+\int_{\Omega_{2}} q_{2, h} \nabla \cdot \mathbf{u}_{2, h} \\
& +\rho \int_{\Omega_{2}}\left(\mathbf{u}_{2, h} \cdot \nabla \mathbf{u}_{2, h}\right) \cdot \mathbf{v}_{2, h},
\end{aligned}
$$

$\mathrm{RR} \mathrm{n}^{\circ} 8415$ 
and the two terms $C_{1}$ and $C_{2}$ are defined by:

$$
\begin{aligned}
C_{1}[\mathbf{u} ;(\mathbf{w}, \mathbf{z}), \mathbf{v}]:= & \frac{\rho}{2} \int_{\Omega_{1}}(\nabla \cdot \mathbf{u}) \mathbf{w} \cdot \mathbf{v}+\frac{\rho}{2} \int_{\Sigma} \mathbf{u} \cdot \mathbf{n}_{1}(\mathbf{z}-\mathbf{w}) \cdot \mathbf{v} \\
C_{2}[(\mathbf{u}, \boldsymbol{\xi}) ;(\mathbf{w}, \mathbf{z}), \mathbf{v}]:= & \frac{\rho}{2} \int_{\Omega_{2}}(\nabla \cdot \mathbf{u}) \mathbf{w} \cdot \mathbf{v}-\frac{\rho}{2} \int_{\Sigma}\left(\mathbf{u} \cdot \mathbf{n}_{2}\right)(\mathbf{w} \cdot \mathbf{v}) \\
& +\frac{\rho}{2} \int_{\Sigma}\left(\boldsymbol{\xi} \cdot \mathbf{n}_{2}\right)(\mathbf{z} \cdot \mathbf{v})
\end{aligned}
$$

The first integral in (8) and (9) is nothing but the standard Temam's trick (see, e.g., [33]). The remaining interface terms are also strongly consistent terms, which are introduced to handle the convective energy contributions related to the discontinuous nature of the discrete solution on the interface. Note that $\mathbf{v}_{1, h}$ and $\mathbf{v}_{2, h}$ are independent, and that $\mathbf{u}_{1, h}$ and $\mathbf{u}_{2, h}$ do not exactly match on the interface.

The last three interface terms of (5) enforce the interface conditions (4) weakly, in a strongly consistent fashion, à la Nitsche. The interface stress term $-\int_{\Sigma} \boldsymbol{\sigma}\left(\mathbf{u}_{2, h}, p_{2, h}\right) \mathbf{n}_{2} \cdot\left(\mathbf{v}_{2, h}-\mathbf{v}_{1, h}\right)$ results from the integration by parts in each subdomain and the application of the relation $(4)_{2}$. The remaining contributions, $\frac{\gamma \mu}{h} \int_{\Sigma}\left(\mathbf{u}_{2, h}-\mathbf{u}_{1, h}\right) \cdot\left(\mathbf{v}_{2, h}-\mathbf{v}_{1, h}\right)$ and $-\int_{\Sigma}\left(\mathbf{u}_{2, h}-\mathbf{u}_{1, h}\right) \cdot \mathbf{n}_{2} q_{2, h}$, are strongly consistent stabilization terms (thanks to $\left.(4)_{1}\right)$ which guarantee the stability of the resulting formulation. The dimensionless parameter $\gamma>0$ is chosen to ensure coercivity (see Lemma 1).

Remark 1 The sixth term in (5), involving the stress tensor on the interface, is computed with the Fluid 2 variables. It could be replaced by $-\int_{\Sigma} \boldsymbol{\sigma}\left(\mathbf{u}_{1, h}, p_{1, h}\right) \mathbf{n}_{1} \cdot\left(\mathbf{v}_{1, h}-\mathbf{v}_{2, h}\right)$, i.e. computed with Fluid 1 variables, without compromising the stability of the method. In this case the seventh term (5) would be $\int_{\Omega_{1}} q_{1, h} \nabla \cdot \mathbf{u}_{1, h}=-\int_{\Sigma}\left(\mathbf{u}_{1, h}-\mathbf{u}_{2, h}\right) \cdot \mathbf{n}_{1} q_{1, h}$. We could also consider a convex combination of both.

\subsubsection{Stability analysis}

The following lemma shows the stability in the energy norm of the semi-discrete problem (5). It makes use of the standard local inverse trace inequality (see [34] e.g.): for all $K \in\left\{\mathcal{T}_{h}\right\}_{h>0}$,

$$
\left\|\mathbf{u}_{h}\right\|_{\partial K}^{2} \leq C_{T} h^{-1}\left\|\mathbf{u}_{h}\right\|_{K}^{2} \quad \forall \mathbf{u}_{h} \in W_{h}
$$

where $\|\cdot\|_{K}$ denotes the $L^{2}(K)$-norm.

Lemma 1 Let $\left(\mathbf{u}_{1, h}, p_{1, h}, \mathbf{u}_{2, h}, p_{2, h}\right)$ be solution of (5). If $\gamma<2 C_{T}$, where $C_{T}>0$ is defined in (10), the following estimate holds

$$
\begin{aligned}
& \frac{\rho}{2}\left(\left\|\mathbf{u}_{1, h}(t)\right\|_{\Omega_{1}}^{2}+\left\|\mathbf{u}_{2, h}(t)\right\|_{\Omega_{2}}^{2}\right)+\frac{3 \mu}{4} \int_{0}^{t}\left(\left\|\boldsymbol{\epsilon}\left(\mathbf{u}_{1, h}\right)\right\|_{\Omega_{1}}^{2}+\left\|\boldsymbol{\epsilon}\left(\mathbf{u}_{2, h}\right)\right\|_{\Omega_{2}}^{2}\right) \\
& \quad+\frac{3 \gamma \mu}{4 h} \int_{0}^{t}\left\|\mathbf{u}_{2, h}-\mathbf{u}_{1, h}\right\|_{\Sigma}^{2} \leq \frac{\rho}{2}\left(\left\|\mathbf{u}_{1, h}(0)\right\|_{\Omega_{1}}^{2}+\left\|\mathbf{u}_{2, h}(0)\right\|_{\Omega_{2}}^{2}\right) .
\end{aligned}
$$


Proof. Taking $\left(\mathbf{v}_{1, h}, q_{1, h}, \mathbf{v}_{2, h}, q_{2, h}\right)=\left(\mathbf{u}_{1, h}, p_{1, h}, \mathbf{u}_{2, h}, p_{2, h}\right)$ in $A_{1}$ and $A_{2}$ yields

$$
\begin{aligned}
A_{1}\left[\mathbf{u}_{1, h} ;\left(\mathbf{u}_{1, h}, p_{1, h}\right),\left(\mathbf{u}_{1, h}, p_{1, h}\right)\right]= & \frac{\rho}{2} \frac{d}{d t}\left\|\mathbf{u}_{1, h}\right\|_{\Omega_{1}}^{2}+2 \mu\left\|\boldsymbol{\epsilon}\left(\mathbf{u}_{1, h}\right)\right\|_{\Omega_{1}}^{2} \\
& -\frac{\rho}{2} \int_{\Omega_{1}}\left(\nabla \cdot \mathbf{u}_{1, h}\right)\left|\mathbf{u}_{1, h}\right|^{2} \\
& +\frac{\rho}{2} \int_{\Sigma}\left(\mathbf{u}_{1, h} \cdot \mathbf{n}_{1, h}\right)\left|\mathbf{u}_{1, h}\right|^{2} \\
A_{2}\left[\mathbf{u}_{2, h} ;\left(\mathbf{u}_{2, h}, p_{2, h}\right),\left(\mathbf{u}_{2, h}, p_{2, h}\right)\right]= & \frac{\rho}{2} \frac{d}{d t}\left\|\mathbf{u}_{2, h}\right\|_{\Omega_{2}}^{2}+2 \mu|| \boldsymbol{\epsilon}\left(\mathbf{u}_{2, h}\right) \|_{\Omega_{2}}^{2} \\
& -\frac{\rho}{2} \int_{\Omega_{2}}\left(\nabla \cdot \mathbf{u}_{2, h}\right)\left|\mathbf{u}_{2, h}\right|^{2} \\
& +\frac{\rho}{2} \int_{\Sigma}\left(\mathbf{u}_{2, h} \cdot \mathbf{n}_{1, h}\right)\left|\mathbf{u}_{2, h}\right|^{2} .
\end{aligned}
$$

In addition, for the terms $C_{1}$ and $C_{2}$ we have

$$
\begin{aligned}
C_{1}\left[\mathbf{u}_{1, h} ;\left(\mathbf{u}_{1, h}, \mathbf{u}_{2, h}\right), \mathbf{u}_{1, h}\right]= & \frac{\rho}{2} \int_{\Omega_{1}}\left(\nabla \cdot \mathbf{u}_{1, h}\right)\left|\mathbf{u}_{1, h}\right|^{2} \\
& +\frac{\rho}{2} \int_{\Sigma}\left(\mathbf{u}_{1, h} \cdot \mathbf{n}_{1}\right)\left(\mathbf{u}_{2, h} \cdot \mathbf{u}_{1, h}-\left|\mathbf{u}_{1, h}\right|^{2}\right), \\
C_{2}\left[\left(\mathbf{u}_{2, h}, \mathbf{u}_{1, h}\right) ;\left(\mathbf{u}_{2, h}, \mathbf{u}_{1, h}\right), \mathbf{u}_{2, h}\right]= & \frac{\rho}{2} \int_{\Omega_{2}}\left(\nabla \cdot \mathbf{u}_{2, h}\right)\left|\mathbf{u}_{2, h}\right|^{2} \\
& -\frac{\rho}{2} \int_{\Sigma}\left(\mathbf{u}_{2, h} \cdot \mathbf{n}_{2}\right)\left|\mathbf{u}_{2, h}\right|^{2} \\
& +\frac{\rho}{2} \int_{\Sigma}\left(\mathbf{u}_{1, h} \cdot \mathbf{n}_{2}\right)\left(\mathbf{u}_{1, h} \cdot \mathbf{u}_{2, h}\right) .
\end{aligned}
$$

Hence by inserting (11)-(14) into (5) we get the following energy equality

$$
\begin{aligned}
& \frac{\rho}{2} \frac{d}{d t}\left(\left\|\mathbf{u}_{1, h}\right\|_{\Omega_{1}}^{2}+\left\|\mathbf{u}_{2, h}\right\|_{\Omega_{2}}^{2}\right)+2 \mu\left(\left\|\boldsymbol{\epsilon}\left(\mathbf{u}_{1, h}\right)\right\|_{\Omega_{1}}^{2}+\left\|\boldsymbol{\epsilon}\left(\mathbf{u}_{2, h}\right)\right\|_{\Omega_{2}}^{2}\right) \\
& \quad+\frac{\gamma \mu}{h}\left\|\mathbf{u}_{2, h}-\mathbf{u}_{1, h}\right\|_{\Sigma}^{2}=2 \mu \int_{\Sigma} \boldsymbol{\epsilon}\left(\mathbf{u}_{2, h}\right) \mathbf{n}_{2} \cdot\left(\mathbf{u}_{2, h}-\mathbf{u}_{1, h}\right) .
\end{aligned}
$$

The right-hand side of (15) is first treated with the Young's inequality and with the local inverse trace inequality (10):

$$
2 \mu \int_{\Sigma} \boldsymbol{\epsilon}\left(\mathbf{u}_{2, h}\right) \mathbf{n}_{2} \cdot\left(\mathbf{u}_{2, h}-\mathbf{u}_{1, h}\right) \leq \frac{4 \mu C_{T}}{\gamma}\left\|\boldsymbol{\epsilon}\left(\mathbf{u}_{2, h}\right)\right\|_{\Omega_{2}}^{2}+\frac{\mu \gamma}{4 h}\left\|\mathbf{u}_{2, h}-\mathbf{u}_{1, h}\right\|_{\Sigma}^{2} .
$$

Using this inequality in (15) and integrating over $(0, t)$, it gives:

$$
\begin{gathered}
\frac{\rho}{2} \quad\left(\left\|\mathbf{u}_{1, h}(t)\right\|_{\Omega_{1}}^{2}+\left\|\mathbf{u}_{2, h}(t)\right\|_{\Omega_{2}}^{2}\right)+2 \mu \int_{0}^{t}\left\|\boldsymbol{\epsilon}\left(\mathbf{u}_{1, h}\right)\right\|_{\Omega_{1}}^{2}+2 \mu\left(1-\frac{2 C_{T}}{\gamma}\right) \int_{0}^{t}\left\|\boldsymbol{\epsilon}\left(\mathbf{u}_{2, h}\right)\right\|_{\Omega_{2}}^{2} \\
+\frac{3 \gamma \mu}{4 h} \int_{0}^{t}\left\|\mathbf{u}_{2, h}-\mathbf{u}_{1, h}\right\|_{\Sigma}^{2} \leq \frac{\rho}{2}\left(\left\|\mathbf{u}_{1, h}(0)\right\|_{\Omega_{1}}^{2}+\left\|\mathbf{u}_{2, h}(0)\right\|_{\Omega_{2}}^{2}\right) .
\end{gathered}
$$

The last result, with condition $\gamma<2 C_{T}$, completes the proof.

$\mathrm{RR} \mathrm{n}^{\circ} 8415$ 


\subsection{Partitioned formulation}

Our goal is to devise an algorithm that yields separate solutions in domain $\Omega_{1}$ and $\Omega_{2}$ via suitable transmission conditions on the interface $\Sigma$. Taking first $\left(\mathbf{v}_{2, h}, q_{2, h}\right)=(\mathbf{0}, 0)$ and then $\left(\mathbf{v}_{1, h}, q_{1, h}\right)$ $=(\mathbf{0}, 0)$, the monolithic formulation (5) can be split in two coupled sub-problems:

1. Fluid sub-problem 1: Given $\left(\mathbf{u}_{2, h}, p_{2, h}\right) \in W_{2, h} \times Q_{2, h}$, find $\left(\mathbf{u}_{1, h}, p_{1, h}\right) \in W_{1, h} \times Q_{1, h}$ such that

$$
\begin{array}{r}
A_{1}\left[\mathbf{u}_{1, h} ;\left(\mathbf{u}_{1, h}, p_{1, h}\right),\left(\mathbf{v}_{1, h}, q_{1, h}\right)\right]+C_{1}\left[\mathbf{u}_{1, h} ;\left(\mathbf{u}_{1, h}, \mathbf{u}_{2, h}\right), \mathbf{v}_{1, h}\right] \\
+\frac{\gamma \mu}{h} \int_{\Sigma}\left(\mathbf{u}_{1, h}-\mathbf{u}_{2, h}\right) \cdot \mathbf{v}_{1, h}+\int_{\Sigma} \boldsymbol{\sigma}\left(\mathbf{u}_{2, h}, p_{2, h}\right) \mathbf{n}_{2} \cdot \mathbf{v}_{1, h}=0
\end{array}
$$

for all $\left(\mathbf{v}_{1, h}, p_{1, h}\right) \in V_{1, h} \times Q_{1, h}$.

2. Fluid sub-problem 2: Given $\left(\mathbf{u}_{1, h}, p_{1, h}\right) \in W_{1, h} \times Q_{1, h}$, find $\left(\mathbf{u}_{2, h}, p_{2, h}\right) \in W_{2, h} \times Q_{2, h}$ such that

$$
\begin{aligned}
& A_{2}\left[\mathbf{u}_{2, h} ;\left(\mathbf{u}_{2, h}, p_{2, h}\right),\left(\mathbf{v}_{2, h}, q_{2, h}\right)\right]+C_{2}\left[\left(\mathbf{u}_{2, h}, \mathbf{u}_{1, h}\right) ;\left(\mathbf{u}_{2, h}, \mathbf{u}_{1, h}\right), \mathbf{v}_{2, h}\right] \\
& \quad+\frac{\gamma \mu}{h} \int_{\Sigma}\left(\mathbf{u}_{2, h}-\mathbf{u}_{1, h}\right) \cdot \mathbf{v}_{2, h}-\int_{\Sigma} \boldsymbol{\sigma}\left(\mathbf{u}_{2, h}, p_{2, h}\right) \mathbf{n}_{2} \cdot \mathbf{v}_{2, h} \\
& \quad-\int_{\Sigma}\left(\mathbf{u}_{2, h}-\mathbf{u}_{1, h}\right) \cdot \mathbf{n}_{2} q_{2, h}=0
\end{aligned}
$$

for all $\left(\mathbf{v}_{2, h}, p_{2, h}\right) \in V_{2, h} \times Q_{2, h}$.

Note that, as in the standard Nitsche method, all the interface terms are evaluated using facewise integration. This partitioned formulation is the basis of the staggered algorithms presented in the next section.

\section{Time-discretization: fully discrete formulations}

The present section is devoted to the time discretization of formulation (5). A backward Euler scheme is used and the non-linear terms are linearized with a standard semi-implicit approach. The time derivatives in (6) and (7) are replaced by $\partial_{\delta t} x^{n}:=\left(x^{n}-x^{n-1}\right) / \delta t$, where $\delta t:=T / N$ denotes the step size, the interval of interest is $(0, T), N \in \mathbb{N}^{+}$is a given integer and $x^{n} \approx x(n \delta t)$ with $0 \leq n \leq N$. The two discrete counterparts of operators $A_{1}$ and $A_{2}$ are defined by:

$$
\begin{aligned}
A_{1, \delta t}\left[\mathbf{u}_{1, h}^{n-1} ;\left(\mathbf{u}_{1, h}^{n}, p_{1, h}^{n}\right),\left(\mathbf{v}_{1, h}, q_{1, h}\right)\right]:= & \rho \int_{\Omega_{1}} \partial_{\delta t} \mathbf{u}_{1, h}^{n} \cdot \mathbf{v}_{1, h}+2 \mu \int_{\Omega_{1}} \boldsymbol{\epsilon}\left(\mathbf{u}_{1, h}^{n}\right): \boldsymbol{\epsilon}\left(\mathbf{v}_{1, h}\right) \\
& -\int_{\Omega_{1}} p_{1, h}^{n} \nabla \cdot \mathbf{v}_{1, h}+\int_{\Omega_{1}} q_{1, h} \nabla \cdot \mathbf{u}_{1, h}^{n} \\
& +\rho \int_{\Omega_{1}} \mathbf{u}_{1, h}^{n-1} \cdot \nabla \mathbf{u}_{1, h}^{n} \cdot \mathbf{v}_{1, h}, \\
A_{2, \delta t}\left[\mathbf{u}_{2, h}^{n-1} ;\left(\mathbf{u}_{2, h}^{n}, p_{2, h}^{n}\right),\left(\mathbf{v}_{2, h}, q_{2, h}\right)\right]:= & \rho \int_{\Omega_{2}} \partial_{\delta t} \mathbf{u}_{2, h}^{n} \mathbf{v}_{2, h}+2 \mu \int_{\Omega_{2}} \boldsymbol{\epsilon}\left(\mathbf{u}_{2, h}^{n}\right): \boldsymbol{\epsilon}\left(\mathbf{v}_{2, h}\right) \\
& -\int_{\Omega_{2}} p_{2, h}^{n} \nabla \cdot \mathbf{v}_{2, h}+\int_{\Omega_{2}} q_{2, h} \nabla \cdot \mathbf{u}_{2, h}^{n} \\
& +\rho \int_{\Omega_{2}} \mathbf{u}_{2, h}^{n-1} \cdot \nabla \mathbf{u}_{2, h}^{n} \cdot \mathbf{v}_{2, h} .
\end{aligned}
$$




\subsection{Implicit coupling}

Before addressing the case of a staggered time-stepping, we start with the study of a monolithic scheme where the two sub-problems are solved simultaneously at each time instant $t^{n}$ : find $\left(\mathbf{u}_{1, h}^{n}, p_{1, h}^{n}, \mathbf{u}_{2, h}^{n}, p_{2, h}^{n}\right) \in W_{1, h} \times Q_{1, h} \times W_{2, h} \times Q_{2, h}$ satisfying the essential boundary conditions and such that

$$
\begin{aligned}
& A_{1, \delta t}\left[\mathbf{u}_{1, h}^{n-1} ;\left(\mathbf{u}_{1, h}^{n}, p_{1, h}^{n}\right),\left(\mathbf{v}_{1, h}, q_{1, h}\right)\right]+A_{2, \delta t}\left[\mathbf{u}_{2, h}^{n-1} ;\left(\mathbf{u}_{2, h}^{n}, p_{2, h}^{n}\right),\left(\mathbf{v}_{2, h}, q_{2, h}\right)\right]+ \\
& +C_{1}\left[\mathbf{u}_{1, h}^{n-1} ;\left(\mathbf{u}_{1, h}^{n}, \mathbf{u}_{2, h}^{n}\right), \mathbf{v}_{1, h}\right]+C_{2}\left[\left(\mathbf{u}_{2, h}^{n-1}, \mathbf{u}_{1, h}^{n-1}\right) ;\left(\mathbf{u}_{2, h}^{n}, \mathbf{u}_{1, h}^{n}\right), \mathbf{v}_{1, h}\right] \\
& +\frac{\gamma \mu}{h} \int_{\Sigma}\left(\mathbf{u}_{2, h}^{n}-\mathbf{u}_{1, h}^{n}\right) \cdot\left(\mathbf{v}_{2, h}-\mathbf{v}_{1, h}\right)-\int_{\Sigma} \boldsymbol{\sigma}\left(\mathbf{u}_{2, h}^{n}, p_{2, h}^{n}\right) \mathbf{n}_{2} \cdot\left(\mathbf{v}_{2, h}-\mathbf{v}_{1, h}\right) \\
& \quad-\int_{\Sigma}\left(\mathbf{u}_{2, h}^{n}-\mathbf{u}_{1, h}^{n}\right) \cdot \mathbf{n}_{2} q_{2, h}=0,
\end{aligned}
$$

for all $\left(\mathbf{v}_{1, h}, q_{1, h}, \mathbf{v}_{2, h}, q_{2, h}\right) \in V_{1, h} \times Q_{1, h} \times V_{2, h} \times Q_{2, h}$.

For the sake of conciseness, the strain rate tensors at time $t^{n}$ are denoted by $\boldsymbol{\epsilon}_{1, h}^{m}:=\boldsymbol{\epsilon}\left(\mathbf{u}_{1, h}^{m}\right)$ and $\boldsymbol{\epsilon}_{2, h}^{m}:=\boldsymbol{\epsilon}\left(\mathbf{u}_{2, h}^{m}\right)$ and the following quantities are introduced:

$$
\begin{aligned}
E^{n}:= & \frac{\rho}{2}\left(\left\|\mathbf{u}_{1, h}^{n}\right\|_{\Omega_{1}}^{2}+\left\|\mathbf{u}_{2, h}^{n}\right\|_{\Omega_{2}}^{2}\right)+\frac{3 \gamma \mu}{4 h} \delta t \sum_{m=1}^{n}\left\|\mathbf{u}_{2, h}^{m}-\mathbf{u}_{1, h}^{m}\right\|_{\Sigma}^{2} \\
& +\frac{2 \mu}{3} \delta t \sum_{m=1}^{n}\left\|\epsilon_{1, h}^{m}\right\|_{\Omega_{1}}^{2}+\frac{2 \mu}{3} \delta t \sum_{m=1}^{n}\left\|\boldsymbol{\epsilon}_{2, h}^{m}\right\|_{\Omega_{2}}^{2}, \\
E^{0}:= & \frac{\rho}{2}\left(\left\|\mathbf{u}_{1, h}^{0}\right\|_{\Omega_{1}}^{2}+\left\|\mathbf{u}_{2, h}^{0}\right\|_{\Omega_{2}}^{2}\right) .
\end{aligned}
$$

The next proposition shows that this monolithic algorithm is stable without any condition on the discretization steps.

Proposition 2 Let $\left(\mathbf{u}_{1, h}^{n}, p_{1, h}^{n}, \mathbf{u}_{2}^{n}, p_{2}^{n}\right)$ be the solution of the monolithic scheme (18). If $\gamma \geq 2 C_{T}$, where $C_{T}>0$ is the constant of the inverse inequality (10), then $E^{n} \leq E^{0}$.

Proof. The proof follows the same steps as the proof of Lemma 1. Choosing $\left(\mathbf{v}_{1, h}, q_{1, h}, \mathbf{v}_{2, h}, q_{2, h}\right)$ $=\left(\mathbf{u}_{1, h}^{n}, p_{1, h}^{n}, \mathbf{u}_{2, h}^{n}, p_{2, h}^{n}\right)$ in (18), the interface terms resulting from the integration by parts of the advection in (16) and (17) cancel with interface stabilization terms (8) and (9). Then summing over $1 \leq m \leq n$ and multiplying by $\delta t$, we obtain:

$$
\begin{aligned}
& \frac{\rho}{2} \sum_{m=1}^{n}\left(\left\|\mathbf{u}_{1, h}^{m}\right\|_{\Omega_{1}}^{2}-\left\|\mathbf{u}_{1, h}^{m-1}\right\|_{\Omega_{1}}^{2}\right)+\frac{\rho}{2} \sum_{m=1}^{n}\left\|\mathbf{u}_{1, h}^{m}-\mathbf{u}_{1, h}^{m-1}\right\|_{\Omega_{1}}^{2}+2 \mu \delta t \sum_{m=1}^{n}\left\|\boldsymbol{\epsilon}_{1, h}^{m}\right\|_{\Omega_{1}}^{2} \\
& \quad+\frac{\rho}{2} \sum_{m=1}^{n}\left(\left\|\mathbf{u}_{2, h}^{m}\right\|_{\Omega_{2}}^{2}-\left\|\mathbf{u}_{2, h}^{m-1}\right\|_{\Omega_{2}}^{2}\right)+\frac{\rho}{2} \sum_{m=1}^{n}\left\|\mathbf{u}_{2, h}^{m}-\mathbf{u}_{2, h}^{m-1}\right\|_{\Omega_{2}}^{2}+2 \mu \delta t \sum_{m=1}^{n}\left\|\boldsymbol{\epsilon}_{2, h}^{m}\right\|_{\Omega_{2}}^{2} \\
& \quad+\frac{\gamma \mu}{h} \delta t \sum_{m=1}^{n}\left\|\mathbf{u}_{2, h}^{m}-\mathbf{u}_{1, h}^{m}\right\|_{\Sigma}^{2} \leq \frac{4 \mu C_{T}}{\gamma} \delta t \sum_{m=1}^{n}\left\|\boldsymbol{\epsilon}_{2, h}^{m}\right\|_{\Omega_{2}}^{2}+\frac{\mu \gamma}{4 h} \delta t \sum_{m=1}^{n}\left\|\mathbf{u}_{2, h}^{m}-\mathbf{u}_{1, h}^{m}\right\|_{\Sigma}^{2},
\end{aligned}
$$

$\mathrm{RR} \mathrm{n}^{\circ} 8415$ 
which can be reformulated as

$$
\begin{aligned}
& \frac{\rho}{2}\left(\left\|\mathbf{u}_{1, h}^{n}\right\|_{\Omega_{1}}^{2}+\left\|\mathbf{u}_{2, h}^{n}\right\|_{\Omega_{2}}^{2}\right)+2 \mu \delta t \sum_{m=1}^{n}\left\|\boldsymbol{\epsilon}_{1, h}^{m}\right\|_{\Omega_{1}}^{2} \\
& +2 \mu\left(1-\frac{2 C_{T}}{\gamma}\right) \delta t \sum_{m=1}^{n}\left\|\boldsymbol{\epsilon}_{2, h}^{m}\right\|_{\Omega_{2}}^{2}+\frac{3 \gamma \mu}{4 h} \delta t \sum_{m=1}^{n}\left\|\mathbf{u}_{2, h}^{m}-\mathbf{u}_{1, h}^{m}\right\|_{\Sigma}^{2} \\
\leq \quad & -\frac{\rho}{2} \delta t\left(\sum_{m=1}^{n}\left\|\mathbf{u}_{1, h}^{m}-\mathbf{u}_{1, h}^{m-1}\right\|_{\Omega_{1}}^{2}+\sum_{m=1}^{n}\left\|\mathbf{u}_{2, h}^{m}-\mathbf{u}_{2, h}^{m-1}\right\|_{\Omega_{2}}^{2}\right) \\
& +\frac{\rho}{2}\left(\left\|\mathbf{u}_{1, h}^{0}\right\|_{\Omega_{1}}^{2}+\left\|\mathbf{u}_{2, h}^{0}\right\|_{\Omega_{2}}^{2}\right),
\end{aligned}
$$

which completes the proof.

\subsection{Explicit coupling: a static pressure formulation}

The monolithic scheme (18) involves the simultaneous computation of $\left(\mathbf{u}_{1, h}^{n}, p_{1, h}^{n}\right)$ and $\left(\mathbf{u}_{2, h}^{n}, p_{2, h}^{n}\right)$. In this section, a first staggered scheme (Algorithm 4.2) is proposed to solve problem (5). This method generalizes the explicit coupling schemes introduced in $[8,10]$ for incompressible fluidstructure interaction.

1. Find $\left(\mathbf{u}_{1, h}^{n}, p_{1, h}^{n}\right) \in W_{1, h} \times Q_{1, h}$ satisfying the essential boundary conditions and such that

$$
\begin{gathered}
A_{1, \delta t}\left[\mathbf{u}_{1, h}^{n-1} ;\left(\mathbf{u}_{1, h}^{n}, p_{1, h}^{n}\right),\left(\mathbf{v}_{1, h}, q_{1, h}\right)\right]+C_{1}\left[\mathbf{u}_{1, h}^{n-1} ;\left(\mathbf{u}_{1, h}^{n}, \mathbf{u}_{2, h}^{n-1}\right), \mathbf{v}_{1, h}\right] \\
+\frac{\gamma \mu}{h} \int_{\Sigma}\left(\mathbf{u}_{1, h}^{n}-\mathbf{u}_{2, h}^{n-1}\right) \cdot \mathbf{v}_{1, h}+\int_{\Sigma} \boldsymbol{\sigma}\left(\mathbf{u}_{2, h}^{n-1}, p_{2, h}^{n-1}\right) \mathbf{n}_{2} \cdot \mathbf{v}_{1, h}=0
\end{gathered}
$$

for all $\left(\mathbf{v}_{1, h}, q_{1, h}\right) \in V_{1, h} \times Q_{1, h}$.

2. Find $\left(\mathbf{u}_{2, h}^{n}, p_{2, h}^{n}\right) \in W_{2, h} \times Q_{2, h}$ satisfying the essential boundary conditions and such that

$$
\begin{aligned}
& A_{2, \delta t}\left[\mathbf{u}_{2, h}^{n-1} ;\left(\mathbf{u}_{2, h}^{n}, p_{2, h}^{n}\right),\left(\mathbf{v}_{2, h}, q_{2, h}\right)\right]+S\left(p_{2, h}^{n}, q_{2, h}\right) \\
& \quad+C_{2}\left[\left(\mathbf{u}_{2, h}^{n-1}, \mathbf{u}_{1, h}^{n-1}\right) ;\left(\mathbf{u}_{2, h}^{n}, \mathbf{u}_{1, h}^{n}\right), \mathbf{v}_{2, h}\right]+\frac{\gamma \mu}{h} \int_{\Sigma}\left(\mathbf{u}_{2, h}^{n}-\mathbf{u}_{1, h}^{n}\right) \cdot \mathbf{v}_{2, h} \\
& -\int_{\Sigma} \boldsymbol{\sigma}\left(\mathbf{u}_{2, h}^{n-1}, p_{2, h}^{n-1}\right) \mathbf{n}_{2} \cdot \mathbf{v}_{2, h}-\int_{\Sigma}\left(\mathbf{u}_{2, h}^{n}-\mathbf{u}_{1, h}^{n}\right) \cdot \mathbf{n}_{2} q_{2, h}=0
\end{aligned}
$$

for all $\left(\mathbf{v}_{2, h}, q_{2, h}\right) \in V_{2, h} \times Q_{2, h}$.

3. Go to next time-step.

As shown in Proposition 3, we are not able to prove its stability. We nevertheless present it because it allows us to introduce the main ideas that will be useful in the next section. In addition, this scheme was stable in most of the numerical simulations where it was tested.

This scheme only yields one solution of each sub-problem at each time step. The terms involving the two forms $A_{1, \delta t}$ and $A_{2, \delta t}$ take the same expressions (16) and (17) as in the monolithic scheme. The differences between the two algorithms only lie in the interface terms. First, the stabilization term $C_{1}\left[\mathbf{u}_{1, h}^{n-1} ;\left(\mathbf{u}_{1, h}^{n}, \mathbf{u}_{2, h}^{n}\right), \mathbf{v}_{1, h}\right]$ of the monolithic algorithm (18) is replaced 
by $C_{1}\left[\mathbf{u}_{1, h}^{n-1} ;\left(\mathbf{u}_{1, h}^{n}, \mathbf{u}_{2, h}^{n-1}\right), \mathbf{v}_{1, h}\right]$ in order to uncouple the two sub-problems. Second, the weakly consistent term

$$
S\left(p_{2, h}^{n}, q_{2, h}\right):=\frac{\gamma_{0} h}{\gamma \mu} \int_{\Sigma}\left(p_{2, h}^{n}-p_{2, h}^{n-1}\right) q_{2, h},
$$

is added in sub-domain $\Omega_{2}$ in order to control spurious pressure fluctuations which appear at the interface because of the explicit coupling between the two sub-domains. The dimensionless parameter $\gamma_{0}>0$ will be fixed to ensure the energy stability of the method.

It is interesting to note that this scheme, obtained from Nitsche's penalty formulation, can be seen as an explicit Robin-Robin scheme associated with the following boundary conditions:

$$
\left\{\begin{array}{c}
\boldsymbol{\sigma}\left(\mathbf{u}_{1}^{n}, p_{1}^{n}\right) \mathbf{n}_{1}+\frac{\gamma \mu}{h} \mathbf{u}_{1}^{n}=\frac{\gamma \mu}{h} \mathbf{u}_{2}^{n-1}-\boldsymbol{\sigma}\left(\mathbf{u}_{2}^{n-1}, p_{2}^{n-1}\right) \mathbf{n}_{2}, \quad \text { on } \quad \Sigma, \\
\boldsymbol{\sigma}\left(\mathbf{u}_{2}^{n}, p_{2}^{n}\right) \mathbf{n}_{2}+\frac{\gamma \mu}{h} \mathbf{u}_{2}^{n}=\frac{\gamma \mu}{h} \mathbf{u}_{1}^{n}+\boldsymbol{\sigma}\left(\mathbf{u}_{2}^{n-1}, p_{2}^{n-1}\right) \mathbf{n}_{2}, \quad \text { on } \quad \Sigma .
\end{array}\right.
$$

We attempt to prove the stability in the energy norm of the explicit formulation (20)-(21). Although the result is not conclusive, it is useful since it pinpoints the difficulty that will be addressed in Algorithm 4.4. In addition, most of the arguments of the proof are reusable for the stability analysis of Algorithm 4.4 .

Proposition 3 Let $\left(\mathbf{u}_{1, h}^{n}, p_{1, h}^{n}, \mathbf{u}_{2, h}^{n}, p_{2, h}^{n}\right)$ be the solution of the staggered scheme (20)-(21). Under the conditions:
a) $\gamma_{0}>4$
b) $\gamma \delta t \leq C_{\Sigma} h$,
c) $\gamma \geq 4 C_{T}$,

where $C_{T}>0$ is the constant of the inverse inequality (10) and $C_{\Sigma}>0$ is given, the following estimate holds:

$$
\begin{aligned}
E^{n}+\frac{\gamma \mu}{2 h} \delta t\left\|\mathbf{u}_{2, h}^{n}\right\|_{\Sigma}^{2}+\frac{\gamma_{0} h}{2 \gamma \mu} \delta t\left\|p_{2, h}^{n}\right\|_{\Sigma}^{2} \leq \quad & 3 E^{0}+\frac{3 C_{\Sigma} \mu}{2}\left\|\mathbf{u}_{2, h}^{0}\right\|_{\Sigma}^{2} \\
& +\frac{3 \gamma_{0} h}{2 \gamma \mu} \delta t\left\|p_{2, h}^{0}\right\|_{\Sigma}^{2}+24 \delta t\left\|\boldsymbol{\epsilon}_{2, h}^{0}\right\|_{\Omega_{2}}^{2} \\
& +\frac{3}{2} \rho \delta t \sum_{m=1}^{n} \int_{\Sigma} \mathbf{u}_{1, h}^{m-1} \cdot \mathbf{n}_{1} \mathbf{u}_{1, h}^{m} \cdot\left(\mathbf{u}_{2, h}^{m}-\mathbf{u}_{2, h}^{m-1}\right) .
\end{aligned}
$$

Proof. Let be $\left(\mathbf{v}_{1, h}, q_{1, h}, \mathbf{v}_{2, h}, q_{2, h}\right)=\left(\mathbf{u}_{1, h}^{n}, p_{1, h}^{n}, \mathbf{u}_{2, h}^{n}, p_{2, h}^{n}\right)$, the two forms $A_{1, \delta t}$ and $A_{2, \delta t}$ give

$$
\begin{aligned}
A_{1, \delta t}\left[\mathbf{u}_{1, h}^{n-1} ;\left(\mathbf{u}_{1, h}^{n}, p_{1, h}^{n}\right),\left(\mathbf{u}_{1, h}^{n}, p_{1, h}^{n}\right)\right]= & \frac{\rho}{2 \delta t}\left(\left\|\mathbf{u}_{1, h}^{n}\right\|_{\Omega_{1}}^{2}-\left\|\mathbf{u}_{1, h}^{n-1}\right\|_{\Omega_{1}}^{2}+\left\|\mathbf{u}_{1, h}^{n}-\mathbf{u}_{1, h}^{n-1}\right\|_{\Omega_{1}}^{2}\right) \\
& +2 \mu\left\|\boldsymbol{\epsilon}_{1, h}^{n}\right\|_{\Omega_{1}}^{2}-\frac{\rho}{2} \int_{\Omega_{1}}\left(\nabla \cdot \mathbf{u}_{1, h}^{n-1}\right)\left|\mathbf{u}_{1, h}^{n}\right|^{2} \\
& +\frac{\rho}{2} \int_{\Sigma}\left(\mathbf{u}_{1, h}^{n-1} \cdot \mathbf{n}_{1, h}\right)\left|\mathbf{u}_{1, h}^{n}\right|^{2}, \\
A_{2, \delta t}\left[\mathbf{u}_{2, h}^{n-1} ;\left(\mathbf{u}_{2, h}^{n}, p_{2, h}^{n}\right),\left(\mathbf{u}_{2, h}^{n}, p_{2, h}^{n}\right)\right]= & \frac{\rho}{2 \delta t}\left(\left\|\mathbf{u}_{2, h}^{n}\right\|_{\Omega_{2}}^{2}-\left\|\mathbf{u}_{2, h}^{n-1}\right\|_{\Omega_{2}}^{2}+\left\|\mathbf{u}_{2, h}^{n}-\mathbf{u}_{2, h}^{n-1}\right\|_{\Omega_{2}}^{2}\right) \\
& +2 \mu\left\|\boldsymbol{\epsilon}_{2, h}^{n}\right\|_{\Omega_{2}}^{2}-\frac{\rho}{2} \int_{\Omega_{2}}\left(\nabla \cdot \mathbf{u}_{2, h}^{n-1}\right)\left|\mathbf{u}_{2, h}^{n}\right|^{2} \\
& +\frac{\rho}{2} \int_{\Sigma}\left(\mathbf{u}_{2, h}^{n-1} \cdot \mathbf{n}_{2, h}\right)\left|\mathbf{u}_{2, h}^{n}\right|^{2} .
\end{aligned}
$$

$\mathrm{RR} \mathrm{n}^{\circ} 8415$ 
Similarly, for the terms $C_{1}$ and $C_{2}$ we get

$$
\begin{aligned}
C_{1}\left[\mathbf{u}_{1, h}^{n-1} ;\left(\mathbf{u}_{1, h}^{n}, \mathbf{u}_{2, h}^{n-1}\right), \mathbf{u}_{1, h}^{n}\right]= & \frac{\rho}{2} \int_{\Omega_{1}}\left(\nabla \cdot \mathbf{u}_{1, h}^{n-1}\right)\left|\mathbf{u}_{1, h}^{n}\right|^{2}-\frac{\rho}{2} \int_{\Sigma}\left(\mathbf{u}_{1, h}^{n-1} \cdot \mathbf{n}_{1}\right)\left|\mathbf{u}_{1, h}^{n}\right|^{2} \\
& +\frac{\rho}{2} \int_{\Sigma}\left(\mathbf{u}_{1, h}^{n-1} \cdot \mathbf{n}_{1}\right)\left(\mathbf{u}_{2, h}^{n-1} \cdot \mathbf{u}_{1, h}^{n}\right), \\
C_{2}\left[\left(\mathbf{u}_{2, h}^{n-1}, \mathbf{u}_{1, h}^{n-1}\right) ;\left(\mathbf{u}_{2, h}^{n}, \mathbf{u}_{1, h}^{n}\right), \mathbf{u}_{2, h}^{n}\right]= & \frac{\rho}{2} \int_{\Omega_{2}}\left(\nabla \cdot \mathbf{u}_{2, h}^{n-1}\right)\left|\mathbf{u}_{2, h}^{n}\right|^{2}-\frac{\rho}{2} \int_{\Sigma}\left(\mathbf{u}_{2, h}^{n-1} \cdot \mathbf{n}_{2}\right)\left|\mathbf{u}_{2, h}^{n}\right|^{2} \\
& +\frac{\rho}{2} \int_{\Sigma}\left(\mathbf{u}_{1, h}^{n-1} \cdot \mathbf{n}_{2}\right)\left(\mathbf{u}_{1, h}^{n} \cdot \mathbf{u}_{2, h}^{n}\right) .
\end{aligned}
$$

By inserting these expressions into (20)-(21), summing over $1 \leq m \leq n$ and multiplying by $\delta t$, we obtain

$$
\begin{aligned}
& \frac{\rho}{2} \sum_{m=1}^{n}\left(\left\|\mathbf{u}_{1, h}^{m}\right\|_{\Omega_{1}}^{2}-\left\|\mathbf{u}_{1, h}^{m-1}\right\|_{\Omega_{1}}^{2}\right)+\frac{\rho}{2} \sum_{m=1}^{n}\left\|\mathbf{u}_{1, h}^{m}-\mathbf{u}_{1, h}^{m-1}\right\|_{\Omega_{1}}^{2}+2 \mu \delta t \sum_{m=1}^{n}\left\|\boldsymbol{\epsilon}_{1, h}^{m}\right\|_{\Omega_{1}}^{2} \\
& +\frac{\rho}{2} \sum_{m=1}^{n}\left(\left\|\mathbf{u}_{2, h}^{m}\right\|_{\Omega_{2}}^{2}-\left\|\mathbf{u}_{2, h}^{m-1}\right\|_{\Omega_{2}}^{2}\right)+\frac{\rho}{2} \sum_{m=1}^{n}\left\|\mathbf{u}_{2, h}^{m}-\mathbf{u}_{2, h}^{m-1}\right\|_{\Omega_{2}}^{2}+2 \mu \delta t \sum_{m=1}^{n}\left\|\boldsymbol{\epsilon}_{2, h}^{m}\right\|_{\Omega_{2}}^{2} \\
& +\frac{\rho}{2} \delta t \sum_{m=1}^{n} \int_{\Sigma} \mathbf{u}_{1, h}^{m-1} \cdot \mathbf{n}_{1} \mathbf{u}_{1, h}^{m} \cdot\left(\mathbf{u}_{2, h}^{m-1}-\mathbf{u}_{2, h}^{m}\right) \\
= & \delta t \sum_{T_{1}}^{\sum_{m=1}^{n} \int_{\Sigma}\left(p_{2, h}^{m}-p_{2, h}^{m-1}\right) \mathbf{n}_{2} \cdot\left(\mathbf{u}_{2, h}^{m}-\mathbf{u}_{1, h}^{m}\right)-\frac{\gamma}{\gamma \mu} \delta t \sum_{m=1}^{n} \int_{\Sigma}\left(p_{2, h}^{m}-p_{2, h}^{m-1}\right) p_{2, h}^{m}} \\
& -\underbrace{\frac{\gamma \mu}{h} \delta t \sum_{m=1}^{n} \int_{\Sigma}\left(\mathbf{u}_{2, h}^{m}-\mathbf{u}_{1, h}^{m}\right) \cdot \mathbf{u}_{2, h}^{m}+\frac{\gamma \mu}{h} \delta t \sum_{m=1}^{n} \int_{\Sigma}\left(\mathbf{u}_{2, h}^{m-1}-\mathbf{u}_{1, h}^{m}\right) \cdot \mathbf{u}_{1, h}^{m}}_{T_{3}} \\
& +\underbrace{2 \mu \delta t \sum_{m=1}^{n} \int_{\Sigma} \boldsymbol{\epsilon}_{2, h}^{m-1} \mathbf{n}_{2} \cdot\left(\mathbf{u}_{2, h}^{m}-\mathbf{u}_{1, h}^{m}\right)}_{T_{2}} .
\end{aligned}
$$

We now proceed by adapting the arguments reported in [8]. Term $T_{1}$ involves the pressure fluctuations at the interface. Applying Young's inequality, it can be written as

$$
\begin{aligned}
T_{1} \leq & \frac{2 h}{\gamma \mu} \delta t \sum_{m=1}^{n}\left\|p_{2, h}^{m}-p_{2, h}^{m-1}\right\|_{\Sigma}^{2}+\frac{\gamma \mu}{8 h} \delta t \sum_{m=1}^{n}\left\|\mathbf{u}_{2, h}^{m}-\mathbf{u}_{1, h}^{m}\right\|_{\Sigma}^{2} \\
& -\frac{1}{2} \frac{\gamma_{0} h}{\gamma \mu} \delta t\left\|p_{2, h}^{n}\right\|_{\Sigma}^{2}+\frac{1}{2} \frac{\gamma_{0} h}{\gamma \mu} \delta t\left\|p_{2, h}^{0}\right\|_{\Sigma}^{2}-\frac{\gamma_{0} h}{\gamma \mu} \frac{\delta t}{2} \sum_{m=1}^{n}\left\|p_{2, h}^{m}-p_{2, h}^{m-1}\right\|_{\Sigma}^{2} \\
= & \frac{\gamma \mu}{8 h} \delta t \sum_{m=1}^{n}\left\|\mathbf{u}_{2, h}^{m}-\mathbf{u}_{1, h}^{m}\right\|_{\Sigma}^{2}-\frac{1}{2} \frac{\gamma_{0} h}{\gamma \mu} \delta t\left\|p_{2, h}^{n}\right\|_{\Sigma}^{2}+\frac{1}{2} \frac{\gamma_{0} h}{\gamma \mu} \delta t\left\|p_{2, h}^{0}\right\|_{\Sigma}^{2} \\
& +\frac{h}{2 \gamma \mu}\left(4-\gamma_{0}\right) \delta t \sum_{m=1}^{n}\left\|p_{2, h}^{m}-p_{2, h}^{m-1}\right\|_{\Sigma}^{2}
\end{aligned}
$$


the last term being negative (assumption of (24a)), it will be removed from the upper bound.

Term $T_{2}$ concerns the velocity fluctuations at the interface. Adding and subtracting $\mathbf{u}_{2, h}^{m}$ in the second integral of $T_{2}$, we have

$$
\begin{aligned}
T_{2}= & -\frac{\gamma \mu}{h} \delta t \sum_{m=1}^{n} \int_{\Sigma}\left(\mathbf{u}_{2, h}^{m}-\mathbf{u}_{1, h}^{m}\right) \cdot \mathbf{u}_{2, h}^{m}+\frac{\gamma \mu}{h} \delta t \sum_{m=1}^{n} \int_{\Sigma}\left(\mathbf{u}_{2, h}^{m-1}-\mathbf{u}_{2, h}^{m}\right) \cdot \mathbf{u}_{1, h}^{m} \\
& +\frac{\gamma \mu}{h} \delta t \sum_{m=1}^{n} \int_{\Sigma}\left(\mathbf{u}_{2, h}^{m}-\mathbf{u}_{1, h}^{m}\right) \cdot \mathbf{u}_{1, h}^{m} \\
= & -\frac{\gamma \mu}{h} \delta t \sum_{m=1}^{n}\left\|\mathbf{u}_{2, h}^{m}-\mathbf{u}_{1, h}^{m}\right\|_{\Sigma}^{2}-\frac{\gamma \mu}{h} \delta t \sum_{m=1}^{n} \int_{\Sigma}\left(\mathbf{u}_{2, h}^{m}-\mathbf{u}_{2, h}^{m-1}\right) \cdot \mathbf{u}_{1, h}^{m} .
\end{aligned}
$$

Adding and subtracting $\mathbf{u}_{2, h}^{m}$ in the second term of this relation, we obtain

$$
\begin{aligned}
- & \frac{\gamma \mu}{h} \delta t \sum_{m=1}^{n} \int_{\Sigma}\left(\mathbf{u}_{2, h}^{m}-\mathbf{u}_{2, h}^{m-1}\right) \cdot \mathbf{u}_{1, h}^{m} \\
= & -\frac{\gamma \mu}{h} \delta t \sum_{m=1}^{n}\left[\int_{\Sigma}\left(\mathbf{u}_{2, h}^{m}-\mathbf{u}_{2, h}^{m-1}\right) \cdot \mathbf{u}_{2, h}^{m}-\int_{\Sigma}\left(\mathbf{u}_{2, h}^{m}-\mathbf{u}_{2, h}^{m-1}\right) \cdot\left(\mathbf{u}_{2, h}^{m}-\mathbf{u}_{1, h}^{m}\right)\right] \\
= & -\frac{\gamma \mu}{h} \delta t \sum_{m=1}^{n}\left[\int_{\Sigma} \frac{\left|\mathbf{u}_{2, h}^{m}\right|^{2}}{2}-\frac{\left|\mathbf{u}_{2, h}^{m-1}\right|^{2}}{2}+\frac{\left|\mathbf{u}_{2, h}^{m}-\mathbf{u}_{2, h}^{m-1}\right|^{2}}{2}\right. \\
& \left.-\int_{\Sigma}\left(\mathbf{u}_{2, h}^{m}-\mathbf{u}_{2, h}^{m-1}\right) \cdot\left(\mathbf{u}_{2, h}^{m}-\mathbf{u}_{1, h}^{m}\right)\right] \\
\leq & -\frac{\gamma \mu}{2 h} \delta t \sum_{m=1}^{n}\left[\left\|\mathbf{u}_{2, h}^{m}\right\|_{\Sigma}^{2}-\left\|\mathbf{u}_{2, h}^{m-1}\right\|_{\Sigma}^{2}-\left\|\mathbf{u}_{2, h}^{m}-\mathbf{u}_{1, h}^{m}\right\|_{\Sigma}^{2}\right] .
\end{aligned}
$$

Using assumption (24b) we finally get

$$
T_{2} \leq-\frac{\gamma \mu}{2 h} \delta t \sum_{m=1}^{n}\left\|\mathbf{u}_{2, h}^{m}-\mathbf{u}_{1, h}^{m}\right\|_{\Sigma}^{2}-\frac{\gamma \mu}{2 h} \delta t\left\|\mathbf{u}_{2, h}^{n}\right\|_{\Sigma}^{2}+\frac{\mu C_{\Sigma}}{2}\left\|\mathbf{u}_{2, h}^{0}\right\|_{\Sigma}^{2}
$$

Applying Young's inequality and the local trace inequality (10), the term $T_{3}$ can be bounded as follows:

$$
\begin{aligned}
T_{3} & \leq \frac{8 \mu C_{T}}{\gamma} \delta t \sum_{m=1}^{n}\left\|\boldsymbol{\epsilon}_{2, h}^{m-1}\right\|_{\Omega_{2}}^{2}+\frac{\gamma \mu}{8 h} \delta t \sum_{m=1}^{n}\left\|\mathbf{u}_{2, h}^{m}-\mathbf{u}_{1, h}^{m}\right\|_{\Sigma}^{2} \\
& =\frac{8 \mu C_{T}}{\gamma} \delta t \sum_{m=1}^{n-1}\left\|\boldsymbol{\epsilon}_{2, h}^{m}\right\|_{\Omega_{2}}^{2}+\frac{8 \mu C_{T}}{\gamma} \delta t\left\|\boldsymbol{\epsilon}_{2, h}^{0}\right\|_{\Omega_{2}}^{2}+\frac{\gamma \mu}{8 h} \delta t \sum_{m=1}^{n}\left\|\mathbf{u}_{2, h}^{m}-\mathbf{u}_{1, h}^{m}\right\|_{\Sigma}^{2} .
\end{aligned}
$$

$\mathrm{RR} \mathrm{n}^{\circ} 8415$ 
Inserting $T_{1}, T_{2}$ and $T_{3}$ in (26), we have:

$$
\begin{aligned}
& \frac{\rho}{2}\left(\left\|\mathbf{u}_{1, h}^{n}\right\|_{\Omega_{1}}^{2}+\left\|\mathbf{u}_{2, h}^{n}\right\|_{\Omega_{2}}^{2}\right)+\frac{\gamma \mu}{4 h} \delta t \sum_{m=1}^{n}\left\|\mathbf{u}_{2, h}^{m}-\mathbf{u}_{1, h}^{m}\right\|_{\Sigma}^{2}+2 \mu \delta t \sum_{m=1}^{n}\left\|\boldsymbol{\epsilon}_{1, h}^{m}\right\|_{\Omega_{1}}^{2} \\
& +2 \mu\left(1-\frac{4 C_{T}}{\gamma}\right) \delta t \sum_{m=1}^{n}\left\|\boldsymbol{\epsilon}_{2, h}^{m}\right\|_{\Omega_{2}}^{2}+\frac{\gamma \mu}{2 h} \delta t\left\|\mathbf{u}_{2, h}^{n}\right\|_{\Sigma}^{2}+\frac{1}{2} \frac{\gamma_{0} h}{\gamma \mu} \delta t\left\|p_{2, h}^{n}\right\|_{\Sigma}^{2} \\
\leq & \frac{\rho}{2}\left(\left\|\mathbf{u}_{1, h}^{0}\right\|_{\Omega_{1}}^{2}+\left\|\mathbf{u}_{2, h}^{0}\right\|_{\Omega_{2}}^{2}\right)+\frac{\mu C_{\Sigma}}{2}\left\|\mathbf{u}_{2, h}^{0}\right\|_{\Sigma}^{2}+\frac{1}{2} \frac{\gamma_{0} h}{\gamma \mu} \delta t\left\|p_{2, h}^{0}\right\|_{\Sigma}^{2}+\frac{8 \mu C_{T}}{\gamma} \delta t\left\|\boldsymbol{\epsilon}_{2, h}^{0}\right\|_{\Omega_{2}}^{2} \\
& +\frac{\rho}{2} \delta t \sum_{m=1}^{n} \int_{\Sigma} \mathbf{u}_{1, h}^{m-1} \cdot \mathbf{n}_{1} \mathbf{u}_{1, h}^{m} \cdot\left(\mathbf{u}_{2, h}^{m}-\mathbf{u}_{2, h}^{m-1}\right) .
\end{aligned}
$$

Finally under the condition (24c) the assertion is proved.

\subsection{Explicit coupling: a stable but inconsistent formulation}

Energy stability can be guaranteed if, instead of $C_{1}$ and $C_{2}$ in Algorithm 4.2, we consider the following alternative terms:

$$
\begin{aligned}
c_{1}\left[\mathbf{u}_{1, h}^{n-1} ; \mathbf{u}_{1, h}^{n}, \mathbf{v}_{1, h}\right] & :=-\frac{\rho}{2} \int_{\Sigma}\left(\mathbf{u}_{1, h}^{n-1} \cdot \mathbf{n}_{1}\right)\left(\mathbf{u}_{1, h}^{n} \cdot \mathbf{v}_{1, h}\right), \\
c_{2}\left[\mathbf{u}_{2, h}^{n-1} ; \mathbf{u}_{2, h}^{n}, \mathbf{v}_{2, h}\right] & :=-\frac{\rho}{2} \int_{\Sigma}\left(\mathbf{u}_{2, h}^{n-1} \cdot \mathbf{n}_{2}\right)\left(\mathbf{u}_{2, h}^{n} \cdot \mathbf{v}_{2, h}\right) .
\end{aligned}
$$

The corresponding staggered scheme is presented in Algorithm 4.3

1. Find $\left(\mathbf{u}_{1, h}^{n}, p_{1, h}^{n}\right) \in W_{1, h} \times Q_{1, h}$ such that

$$
\begin{aligned}
& A_{1, \delta t}\left[\mathbf{u}_{1, h}^{n-1} ;\left(\mathbf{u}_{1, h}^{n}, p_{1, h}^{n}\right)\left(\mathbf{v}_{1, h}, q_{1, h}\right)\right]+c_{1}\left[\mathbf{u}_{1, h}^{n-1} ; \mathbf{u}_{1, h}^{n}, \mathbf{v}_{1, h}\right] \\
& \quad+\frac{\gamma \mu}{h} \int_{\Sigma}\left(\mathbf{u}_{1, h}^{n}-\mathbf{u}_{2, h}^{n-1}\right) \cdot \mathbf{v}_{1, h}+\int_{\Sigma} \boldsymbol{\sigma}\left(\mathbf{u}_{2, h}^{n-1}, p_{2, h}^{n-1}\right) \mathbf{n}_{2} \cdot \mathbf{v}_{1, h}=0
\end{aligned}
$$

for all $\left(\mathbf{v}_{1, h}, q_{1, h}\right) \in V_{1, h} \times Q_{1, h}$.

2. Find $\left(\mathbf{u}_{2, h}^{n}, p_{2, h}^{n}\right) \in W_{2, h} \times Q_{2, h}$ such that

$$
\begin{aligned}
& A_{2, \delta t}\left[\mathbf{u}_{2, h}^{n-1} ;\left(\mathbf{u}_{2, h}^{n}, p_{2, h}^{n}\right)\left(\mathbf{v}_{2, h}, q_{1, h}\right)\right]+S\left(p_{2, h}^{n}, q_{2, h}\right) \\
& +c_{2}\left[\mathbf{u}_{2, h}^{n-1} ; \mathbf{u}_{2, h}^{n}, \mathbf{v}_{2, h}\right]+\frac{\gamma \mu}{h} \int_{\Sigma}\left(\mathbf{u}_{2, h}^{n}-\mathbf{u}_{1, h}^{n}\right) \cdot \mathbf{v}_{2, h} \\
& -\int_{\Sigma} \boldsymbol{\sigma}\left(\mathbf{u}_{2, h}^{n-1}, p_{2, h}^{n-1}\right) \mathbf{n}_{2} \cdot \mathbf{v}_{2, h}-\int_{\Sigma}\left(\mathbf{u}_{2, h}^{n}-\mathbf{u}_{1, h}^{n}\right) \cdot \mathbf{n}_{2} q_{2, h}=0
\end{aligned}
$$

for all $\left(\mathbf{v}_{2, h}, q_{2, h}\right) \in V_{2, h} \times Q_{2, h}$.

3. Go to next time-step.

Note that this amounts to consider the skew-symmetric formulation of the convective term 
in each sub-domain, namely,

$$
\frac{1}{2} \int_{\Omega_{i}} \mathbf{u}_{i} \cdot \nabla \mathbf{u}_{i} \cdot \mathbf{v}_{i}-\frac{1}{2} \int_{\Omega_{i}} \mathbf{u}_{i} \cdot \nabla \mathbf{v}_{i} \cdot \mathbf{u}_{i} .
$$

Indeed, using integration by parts we have

$$
\frac{1}{2} \int_{\Omega_{i}} \mathbf{u}_{i} \cdot \nabla \mathbf{u}_{i} \cdot \mathbf{v}_{i}-\frac{1}{2} \int_{\Omega_{i}} \mathbf{u}_{i} \cdot \nabla \mathbf{v}_{i} \cdot \mathbf{u}_{i}=\int_{\Omega_{i}} \mathbf{u}_{i} \cdot \nabla \mathbf{u}_{i} \cdot \mathbf{v}_{i}-\frac{1}{2} \int_{\Sigma}\left(\mathbf{u}_{i} \cdot \mathbf{n}_{i}\right)\left(\mathbf{u}_{i} \cdot \mathbf{v}_{i}\right) .
$$

A straightforward adaptation of Proposition 3 hence shows that Algorithm 4.3 is energy stable.

The skew-symmetrized form (29) is commonly used in the staggered time-marching of fluidfluid interaction models based on rigid-lid coupling condition conditions (see, e.g., [14] and the references therein), for which $\mathbf{u}_{i} \cdot \mathbf{n}_{i}=0$ on $\Sigma$. The expression (29) is thus consistent, since the interface term in the right-hand side of (30) vanishes. Clearly, this does not hold for the coupled problem (2)-(4) and, therefore, Algorithm 4.3 involves an inconsistent treatment of (4). In fact, the following (inconsistent) Robin-Robin splitting is enforced

$$
\left\{\begin{array}{c}
\boldsymbol{\sigma}\left(\mathbf{u}_{1}^{n}, p_{1}^{n}\right) \mathbf{n}_{1}-\frac{1}{2}\left(\mathbf{u}_{1}^{n-1} \cdot \mathbf{n}_{1}\right) \mathbf{u}_{1}^{n}+\frac{\gamma \mu}{h} \mathbf{u}_{1}^{n}=\frac{\gamma \mu}{h} \mathbf{u}_{2}^{n-1}-\boldsymbol{\sigma}\left(\mathbf{u}_{2}^{n-1}, p_{2}^{n-1}\right) \mathbf{n}_{2}, \quad \text { on } \quad \Sigma, \\
\boldsymbol{\sigma}\left(\mathbf{u}_{2}^{n}, p_{2}^{n}\right) \mathbf{n}_{2}-\frac{1}{2}\left(\mathbf{u}_{2}^{n-1} \cdot \mathbf{n}_{2}\right) \mathbf{u}_{2}^{n}+\frac{\gamma \mu}{h} \mathbf{u}_{2}^{n}=\frac{\gamma \mu}{h} \mathbf{u}_{1}^{n}+\boldsymbol{\sigma}\left(\mathbf{u}_{2}^{n-1}, p_{2}^{n-1}\right) \mathbf{n}_{2}, \quad \text { on } \quad \Sigma .
\end{array}\right.
$$

The numerical experiments reported in Section 5 confirm the expected poor accuracy of this method.

\subsection{Explicit coupling: a total pressure formulation}

We propose in this Section a provably stable staggered algorithm. The difficulty to establish the stability of Algorithm 4.2 came from the last term of (25) which resulted from the integration by parts of the advection. The get rid of this term, we suggest to use a formulation for which the advection cancels in the energy equation without any integration by parts. This can be achieved by introducing the total pressure $\pi:=p+\rho \frac{\left|\mathbf{u}^{2}\right|}{2}$ and by reformulating the Navier-Stokes equation as:

$$
\begin{aligned}
& \rho \partial_{t} \mathbf{u}+\rho \mathbf{u} \cdot \nabla \mathbf{u}-\rho(\nabla \mathbf{u})^{\mathrm{T}} \mathbf{u}-\nabla \cdot \boldsymbol{\sigma}(\mathbf{u}, \pi)=0, \quad \text { in } \Omega, \\
& \nabla \cdot \mathbf{u}=0, \quad \text { in } \Omega, \\
& \mathbf{u}=0, \quad \text { on } \partial \Omega \text {. }
\end{aligned}
$$

This formulation was in particular discussed in [24, page 337]. It simply results from the relation:

$$
-\nabla \cdot \boldsymbol{\sigma}(\mathbf{u}, p)=-\frac{\rho}{2} \nabla|\mathbf{u}|^{2}-\nabla \cdot \boldsymbol{\sigma}(\mathbf{u}, \pi)=-\rho(\nabla \mathbf{u})^{\mathrm{T}} \mathbf{u}-\nabla \cdot \boldsymbol{\sigma}(\mathbf{u}, \pi) .
$$

Note that the pressure unknown is now the total pressure $\pi$. This is not the case of the curl $\mathbf{u} \times \mathbf{u}$ formulation used in many works to enforce the total pressure in the boundary conditions, but which keeps the static pressure $p$ as unknown (see, e.g., [3, 17, 25, 31, 35]). The variational formulation derived from (31) naturally leads to replace the two forms $A_{1, \delta t}$ and $A_{2, \delta t}$ used in the Algorithm 4.2 by the new forms $A_{1, \delta t}^{\pi}$ and $A_{2, \delta t}^{\pi}$ defined by

$$
\begin{aligned}
& A_{1, \delta t}^{\pi}\left[\mathbf{u}_{1, h}^{n-1} ;\left(\mathbf{u}_{1, h}^{n}, \pi_{1, h}^{n}\right),\left(\mathbf{v}_{1, h}, q_{1, h}\right)\right]:=\rho \int_{\Omega_{1}} \partial_{\delta t} \mathbf{u}_{1, h}^{n} \cdot \mathbf{v}_{1, h}+2 \mu \int_{\Omega_{1}} \boldsymbol{\epsilon}\left(\mathbf{u}_{1, h}^{n}\right): \boldsymbol{\epsilon}\left(\mathbf{v}_{1, h}\right) \\
& +\rho \int_{\Omega_{1}}\left(\left(\mathbf{u}_{1, h}^{n} \cdot \nabla \mathbf{u}_{1, h}^{n-1}\right) \cdot \mathbf{v}_{1, h}-\left(\mathbf{v}_{1, h} \cdot \nabla \mathbf{u}_{1, h}^{n-1}\right) \cdot \mathbf{u}_{1, h}^{n}\right)-\int_{\Omega_{1}} \pi_{1, h}^{n} \nabla \cdot \mathbf{v}_{1, h}+\int_{\Omega_{1}} q_{1, h} \nabla \cdot \mathbf{u}_{1, h}^{n},
\end{aligned}
$$




$$
\begin{aligned}
& A_{2, \delta t}^{\pi}\left[\mathbf{u}_{2, h}^{n-1} ;\left(\mathbf{u}_{2, h}^{n}, \pi_{2, h}^{n}\right),\left(\mathbf{v}_{2, h}, q_{2, h}\right)\right]:=\rho \int_{\Omega_{2}} \partial_{\delta t} \mathbf{u}_{2, h}^{n} \mathbf{v}_{2, h}+2 \mu \int_{\Omega_{2}} \boldsymbol{\epsilon}\left(\mathbf{u}_{2, h}^{n}\right): \boldsymbol{\epsilon}\left(\mathbf{v}_{2, h}\right) \\
& +\rho \int_{\Omega_{2}}\left(\left(\mathbf{u}_{2, h}^{n} \cdot \nabla \mathbf{u}_{2, h}^{n-1}\right) \cdot \mathbf{v}_{2, h}-\left(\mathbf{v}_{2, h} \cdot \nabla \mathbf{u}_{2, h}^{n-1}\right) \cdot \mathbf{u}_{2, h}^{n}\right)-\int_{\Omega_{2}} \pi_{2, h}^{n} \nabla \cdot \mathbf{v}_{2, h}+\int_{\Omega_{2}} q_{2, h} \nabla \cdot \mathbf{u}_{2, h}^{n} .
\end{aligned}
$$

Note that with this new formulation, the last integral in (6) and (7) (Temam's trick) is no longer necessary. The new staggered scheme is presented in Algorithm 4.4.

1. Find $\left(\mathbf{u}_{1, h}^{n}, \pi_{1, h}^{n}\right) \in W_{1, h} \times Q_{1, h}$ satisfying the essential boundary conditions and such that

$$
\begin{aligned}
& A_{1, \delta t}^{\pi}\left[\mathbf{u}_{1, h}^{n-1} ;\left(\mathbf{u}_{1, h}^{n}, \pi_{1, h}^{n}\right),\left(\mathbf{v}_{1, h}, q_{1, h}\right)\right]+\frac{\gamma \mu}{h} \int_{\Sigma}\left(\mathbf{u}_{1, h}^{n}-\mathbf{u}_{2, h}^{n-1}\right) \cdot \mathbf{v}_{1, h} \\
& \quad+\int_{\Sigma} \boldsymbol{\sigma}\left(\mathbf{u}_{2, h}^{n-1}, \pi_{2, h}^{n-1}\right) \mathbf{n}_{2} \cdot \mathbf{v}_{1, h}=0
\end{aligned}
$$

for all $\left(\mathbf{v}_{1, h}, q_{1, h}\right) \in V_{1, h} \times Q_{1, h}$.

2. Find $\left(\mathbf{u}_{2, h}^{n}, \pi_{2, h}^{n}\right) \in W_{2, h} \times Q_{2, h}$ satisfying the essential boundary conditions and such that

$$
\begin{aligned}
& A_{2, \delta t}^{\pi}\left[\mathbf{u}_{2, h}^{n-1} ;\left(\mathbf{u}_{2, h}^{n}, \pi_{2, h}^{n}\right),\left(\mathbf{v}_{2, h}, q_{2, h}\right)\right]+S\left(\pi_{2, h}^{n}, q_{2, h}\right) \\
& +\frac{\gamma \mu}{h} \int_{\Sigma}\left(\mathbf{u}_{2, h}^{n}-\mathbf{u}_{1, h}^{n}\right) \cdot \mathbf{v}_{2, h}-\int_{\Sigma} \boldsymbol{\sigma}\left(\mathbf{u}_{2, h}^{n-1}, \pi_{2, h}^{n-1}\right) \mathbf{n}_{2} \cdot \mathbf{v}_{2, h} \\
& \quad-\int_{\Sigma}\left(\mathbf{u}_{2, h}^{n}-\mathbf{u}_{1, h}^{n}\right) \cdot \mathbf{n}_{2} q_{2, h}=0
\end{aligned}
$$

for all $\left(\mathbf{v}_{2, h}, q_{2, h}\right) \in V_{2, h} \times Q_{2, h}$.

3. Go to next time-step.

As with the previous formulation, the pressure fluctuation at the interface are controlled by the term $S\left(\pi_{2, h}^{n}, q_{2, h}\right)$ in sub-domain 2. Again, the two sub-problems can be seen as coupled through the Robin-Robin transmission conditions:

$$
\left\{\begin{aligned}
\boldsymbol{\sigma}\left(\mathbf{u}_{1}^{n}, \pi_{1}^{n}\right) \mathbf{n}_{1}+\frac{\gamma \mu}{h} \mathbf{u}_{1}^{n}=\frac{\gamma \mu}{h} \mathbf{u}_{2}^{n-1}-\boldsymbol{\sigma}\left(\mathbf{u}_{2}^{n-1}, \pi_{2}^{n-1}\right) \mathbf{n}_{2}, & \text { on } \quad \Sigma, \\
\boldsymbol{\sigma}\left(\mathbf{u}_{2}^{n}, \pi_{2}^{n}\right) \mathbf{n}_{2}+\frac{\gamma \mu}{h} \mathbf{u}_{2}^{n}=\frac{\gamma \mu}{h} \mathbf{u}_{1}^{n}+\boldsymbol{\sigma}\left(\mathbf{u}_{2}^{n-1}, \pi_{2}^{n-1}\right) \mathbf{n}_{2}, & \text { on } \quad \Sigma .
\end{aligned}\right.
$$

This is similar to (23), but the static pressure has been replaced by the total pressure.

Remark 2 The total pressure formulation can be obviously used in the implicit case. The proof of the stability analysis is performed in the same way as in Proposition 2, with the total pressure variable $\pi_{i, h}$ instead of $p_{i, h}, i=1,2$ but without the interface stabilization terms (8) and (9).

Remark 3 If the proposed splitting schemes were used within a fluid-structure interaction framework, the fluid stress on the fluid-solid interface could not be directly obtained as the residual of the fluid variational formulation. Instead, this interface load should be explicitly computed via face-wise integration.

The next proposition shows that Algorithm 4.4 is conditionally stable in the energy norm. 
Proposition 4 Let $\left(\mathbf{u}_{1, h}^{n}, \pi_{1, h}^{n}, \mathbf{u}_{2, h}^{n}, \pi_{2, h}^{n}\right)$ be the solution of (34)-(35). Under the conditions (24), there holds:

$$
\begin{aligned}
E^{n}+\frac{\gamma \mu}{2 h} \delta t\left\|\mathbf{u}_{2, h}^{n}\right\|_{\Sigma}^{2}+\frac{1}{2} \frac{\gamma_{0} h}{\gamma \mu} \delta t\left\|\pi_{2, h}^{n}\right\|_{\Omega_{2}}^{2} \leq & 3 E^{0}+\frac{3}{2} C_{\Sigma} \mu\left\|\mathbf{u}_{2, h}^{0}\right\|_{\Sigma}^{2} \\
& +\frac{3}{2} \frac{\gamma_{0} h}{\gamma \mu} \delta t\left\|\pi_{2, h}^{0}\right\|_{\Sigma}^{2}+24 \delta t\left\|\boldsymbol{\epsilon}_{2, h}^{0}\right\|_{\Omega_{2}}^{2}
\end{aligned}
$$

where $E^{n}, n \geq 1$, and $E^{0}$ are defined in (19) and (20).

Proof. Taking $\left(\mathbf{v}_{1, h}, q_{1, h}, \mathbf{v}_{2, h}, q_{2, h}\right)=\left(\mathbf{u}_{1, h}^{n}, \pi_{1, h}^{n}, \mathbf{u}_{2, h}^{n}, \pi_{2, h}^{n}\right)$, the terms related to advection in (32) and (33) cancel each others. Therefore, multiplying for $\delta t$ and summing over $1 \leq m \leq n$, the system (34)-(35) can be written as

$$
\begin{aligned}
& \frac{\rho}{2} \sum_{m=1}^{n}\left(\left\|\mathbf{u}_{1, h}^{m}\right\|_{\Omega_{1}}^{2}-\left\|\mathbf{u}_{1, h}^{m-1}\right\|_{\Omega_{1}}^{2}\right)+\frac{\rho}{2} \sum_{m=1}^{n}\left\|\mathbf{u}_{1, h}^{m}-\mathbf{u}_{1, h}^{m-1}\right\|_{\Omega_{1}}^{2}+2 \mu \delta t \sum_{m=1}^{n}\left\|\boldsymbol{\epsilon}_{1, h}^{m}\right\|_{\Omega_{1}}^{2} \\
& +\frac{\rho}{2} \sum_{m=1}^{n}\left(\left\|\mathbf{u}_{2, h}^{m}\right\|_{\Omega_{2}}^{2}-\left\|\mathbf{u}_{2, h}^{m-1}\right\|_{\Omega_{2}}^{2}\right)+\frac{\rho}{2} \sum_{m=1}^{n}\left\|\mathbf{u}_{2, h}^{m}-\mathbf{u}_{2, h}^{m-1}\right\|_{\Omega_{2}}^{2}+2 \mu \delta t \sum_{m=1}^{n}\left\|\boldsymbol{\epsilon}_{2, h}^{m}\right\|_{\Omega_{2}}^{2} \\
& =\underbrace{\delta t \sum_{m=1}^{n} \int_{\Sigma}\left(\pi_{2, h}^{m}-\pi_{2, h}^{m-1}\right) \mathbf{n}_{2} \cdot\left(\mathbf{u}_{2, h}^{m}-\mathbf{u}_{1, h}^{m}\right)-\frac{\gamma_{0} h}{\gamma \mu} \delta t \sum_{m=1}^{n} \int_{\Sigma}\left(\pi_{2, h}^{m}-\pi_{2, h}^{m-1}\right) \pi_{2, h}^{m}}_{T_{1}} \\
& -\underbrace{\frac{\gamma \mu}{h} \delta t \sum_{m=1}^{n} \int_{\Sigma}\left(\mathbf{u}_{2, h}^{m}-\mathbf{u}_{1, h}^{m}\right) \cdot \mathbf{u}_{2, h}^{m}+\frac{\gamma \mu}{h} \delta t \sum_{m=1}^{n} \int_{\Sigma}\left(\mathbf{u}_{2, h}^{m-1}-\mathbf{u}_{1, h}^{m}\right) \cdot \mathbf{u}_{1, h}^{m}}_{T_{2}} \\
& +\underbrace{2 \mu \delta t \sum_{m=1}^{n} \int_{\Sigma} \boldsymbol{\epsilon}_{2, h}^{m-1} \mathbf{n}_{2} \cdot\left(\mathbf{u}_{2, h}^{m}-\mathbf{u}_{1, h}^{m}\right)}_{T_{3}} .
\end{aligned}
$$

The terms in the right-hand side are bounded mutatis mutandis as in the proof of Proposition 3. This completes the proof.

\section{$5 \quad$ Numerical Examples}

In this section, we first discuss some tests to illustrate the influence of the stabilization parameters $\gamma$ and $\gamma_{0}$, then we concentrate on hemodynamics applications. For comparison purposes, the following error indicators will be used:

$$
\varepsilon_{x_{i}}:=\frac{\left\|x_{i}-x_{\mathrm{ref}}\right\|_{L^{2}(\Gamma)}}{\left\|x_{\mathrm{ref}}\right\|_{L^{2}(\Gamma)}}, \quad \varepsilon_{x}:=\frac{\left\|x_{1}-x_{2}\right\|_{L^{2}(\Sigma)}}{\left\|x_{\mathrm{ref}}\right\|_{L^{2}(\Sigma)}} .
$$

The first indicator measures the relative error on a part of the boundary $\Gamma \subset \partial \Omega_{i}$ for the physical quantity $x_{i}$ (defined in $\Omega_{i}$ ), $i=1,2$. The second indicator gives the relative interface drop of the variable $x$ across the interface $\Sigma$. The subscript ref indicates a quantity from the reference solution, obtained by solving problems (1) or (31) in the whole domain $\Omega$, with a standard conforming finite element method and a semi-implicit monolithic time-stepping. 


\subsection{Impact of the stabilization parameters}

In this paragraph we investigate the impact of the stabilization parameters $\gamma$ and $\gamma_{0}$ on the accuracy of Algorithm 4.2. We consider a two coupled Stokes problems in a rectangular domain $[0,3] \times[0,3] \cup[3,6] \times[0,3]$, all the space units are in $\mathrm{cm}$. Note that in this case, only the interface pressure stabilization (22) is considered, since the convective non-linear terms are neglected. A constant velocity $(400,0)^{\mathrm{T}} \mathrm{cm} / \mathrm{s}$ is imposed on the left boundary and zero traction on the right boundary. A no-slip condition is enforced on the upper and lower sides. The density $\rho=1.06 \mathrm{~g} / \mathrm{cm}^{3}$ and the dynamic viscosity $\mu=0.04$ poise are those typically encountered in blood flow simulations. The spatial discretization is based on $P_{1} / P_{1}$ stabilized finite elements. The time-step is $\tau=10^{-4} \mathrm{~s}$ and the space step is $h=0.05 \mathrm{~cm}$. The tests are run with $\gamma_{0}=0$, $1,9,16$ and $\gamma=25,250,2500,5000$. All the numerical computations have been performed in FreeFem ++ (see [23]).

In Figure 1, the relative error on the outlet velocity and on the pressure drop at the interface are plotted. These results suggest that the optimal choice of the parameters is $\gamma=2500$ and $\gamma_{0}=1$, which gives a good compromise between the velocity and pressure errors (in particular $\varepsilon_{\mathbf{u}_{2}}=0.0873$ and $\left.\varepsilon_{p}=0.0953\right)$. The values for $\gamma_{0}=0$ are not reported in Figure 1 since the corresponding numerical solution is unstable. This highlights the importance of the interface pressure stabilization (22).
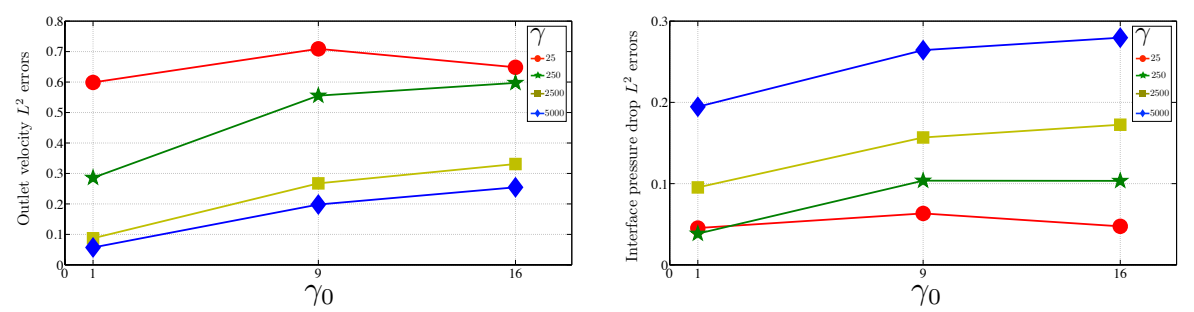

Figure 1: Outlet velocity errors $\varepsilon_{\mathbf{u}_{2}}$ (left) and interface pressure drop errors $\varepsilon_{p}$ (right).

The last 2D-test is performed in the same rectangular domain with the two optimal values of $\gamma$ and $\gamma_{0}$ but neglecting the last term of (21), i.e. the consistent term that control the pressure contribution of the stress on the interface. In this case the outlet velocity error becomes $\varepsilon_{\mathbf{u}_{2}}=0.1319$ and the interface pressure drop error is now $\varepsilon_{p}=0.1082$. Even if the result remains stable, without considering this term an increasing of the errors can be noticed.

\subsection{Aortic blood flow simulation}

The computational domain is the idealized geometry reported in Figure 2 (left), including the aortic root, with the valve, and the aortic arch. The two-domain partitioning is shown in Figure 2 (middle). This geometrical splitting is motivated by the fact that, in order to describe the blood dynamics through the valve, different modeling options can be incorporated within the aortic root $\Omega_{1}$ (see, e.g., $[1,15]$ ). Here, for simplicity, the aortic valve is frozen in its open configuration. No-slip boundary conditions are imposed on the three leaflets of the valve.

A sinusoidal waveform, see Figure 2 (right), is imposed on the inlet boundary $\Gamma_{i n}$. The systolic phase, corresponding to the first half of the cardiac cycle, delivers a maximum flux of about $235 \mathrm{~cm}^{3} / \mathrm{s}$. In the second half (diastolic phase), the inlet flow is set to zero. The resulting cardiac output is approximatively $4.5 \mathrm{dm}^{3} / \mathrm{min}$. The outlets $\Gamma_{a}, \Gamma_{b}$ and $\Gamma_{c}$ correspond to the brachiocephalic artery, left common carotid and subclavian artery, respectively. The outlet $\Gamma_{\text {out }}$ 

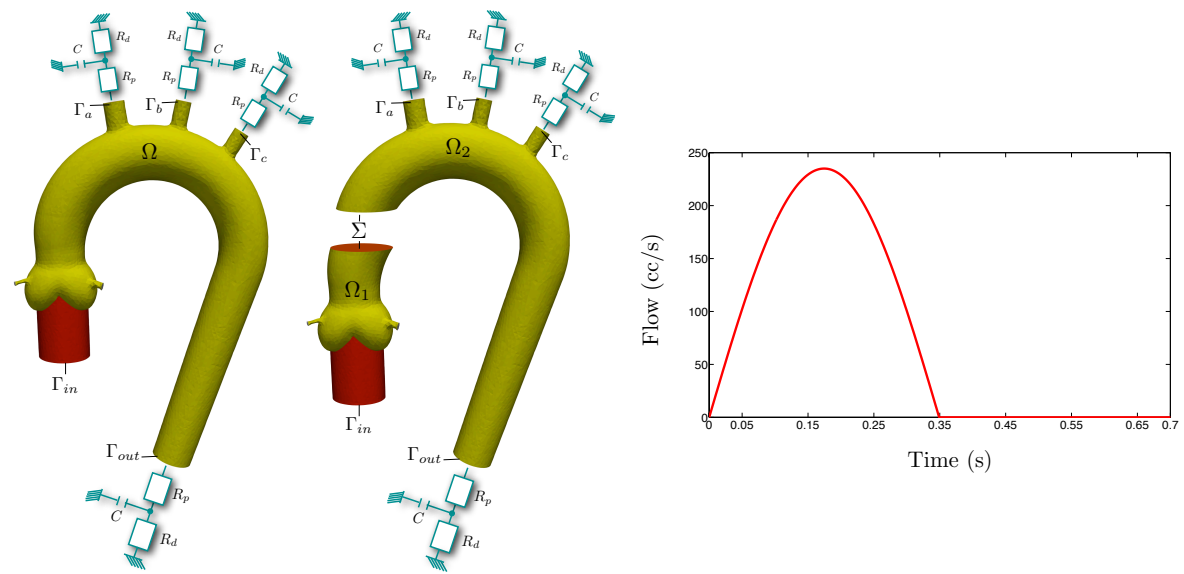

Figure 2: Reference domain (left), sub-domains definition (middle) and inflow waveform (right).

Table 1: Windkessel parameters.

\begin{tabular}{|c|c|c|c|c|}
\cline { 2 - 5 } \multicolumn{1}{c|}{} & $\Gamma_{a}$ & $\Gamma_{b}$ & $\Gamma_{c}$ & $\Gamma_{\text {out }}$ \\
\hline$R_{p}\left(\right.$ dyn $\left.\cdot s \cdot \mathrm{cm}^{-5}\right)$ & $0.05 \times 10^{4}$ & $0.19 \times 10^{4}$ & $0.075 \times 10^{4}$ & $0.015 \times 10^{4}$ \\
\hline$R_{d}\left(\right.$ dyn $\left.\cdot s \cdot \mathrm{cm}^{-5}\right)$ & $0.85 \times 10^{4}$ & $3.22 \times 10^{4}$ & $1.25 \times 10^{4}$ & $0.25 \times 10^{4}$ \\
\hline$C\left(\mathrm{~cm}^{5} \cdot\right.$ dyn $\left.^{-1}\right)$ & $0.95 \times 10^{-4}$ & $0.25 \times 10^{-4}$ & $0.64 \times 10^{-4}$ & $3.17 \times 10^{-4}$ \\
\hline
\end{tabular}

is located in the descending aorta. The neglected portions of the circulation are taken into account by imposing the natural boundary condition $\boldsymbol{\sigma} \mathbf{n}=-P_{p} \mathbf{n}$ on each outlet. The proximal pressure $P_{p}$ is described by a (0D) three-element Windkessel model (see, e.g., [4, 28]) given by the ODE:

$$
C R_{d} \frac{\mathrm{d} P_{p}}{\mathrm{~d} t}+P_{p}=Q\left(R_{d}+R_{p}\right)+C R_{p} R_{d} \frac{\mathrm{d} Q}{\mathrm{~d} t},
$$

where $Q$ stands for the outlet flow rate, $R_{p}$ and $R_{d}$ denote the proximal and distal resistances and $C$ is the capacitance representing the compliance of the blood vessels. The Windkessel parameters are reported in Table 1. For simplicity, the left and right coronaries, located in the aortic sinus, are closed. The physical parameters for blood are chosen as $\rho=1.06 \mathrm{~g} / \mathrm{cm}^{3}$ and $\mu=0.04$ poise. The space discretization is based on $P_{1} / P_{1}$ finite elements, stabilized with the SUPG/PSPG method. The stabilization parameters are $\gamma=2500$ and $\gamma_{0}=1$. For comparison purposes, the reference solution has been generated by solving the Navier-Stokes equations associated in the whole domain $\Omega$. The meshes of $\Omega, \Omega_{1}$ and $\Omega_{2}$ are, respectively, made of 177651,53960 and 126200 tetrahedra. Six cardiac cycles have been simulated, using a time-step length of $\tau=10^{-3} s$.

\subsubsection{Static pressure formulations}

In this paragraph the results obtained with Algorithms 4.2 and 4.3 are compared with the reference solution, obtained by solving (1) with a standard conforming finite element method and a semi-implicit monolithic time-stepping. Figure 3 displays the snapshots of the velocity magnitude at two time instants of the third cardiac cycle: $t=1.575 \mathrm{~s}$ middle of systole (top), and $t=1.750 \mathrm{~s}$ beginning of diastole (bottom). The solution obtained with Algorithm 4.2 is close to the reference solution on the interface and on the outlets. Note that even if we were not able to prove its energy stability (Proposition 3), the simulation is stable in this test case. 
(a)

(b)

$(c)$
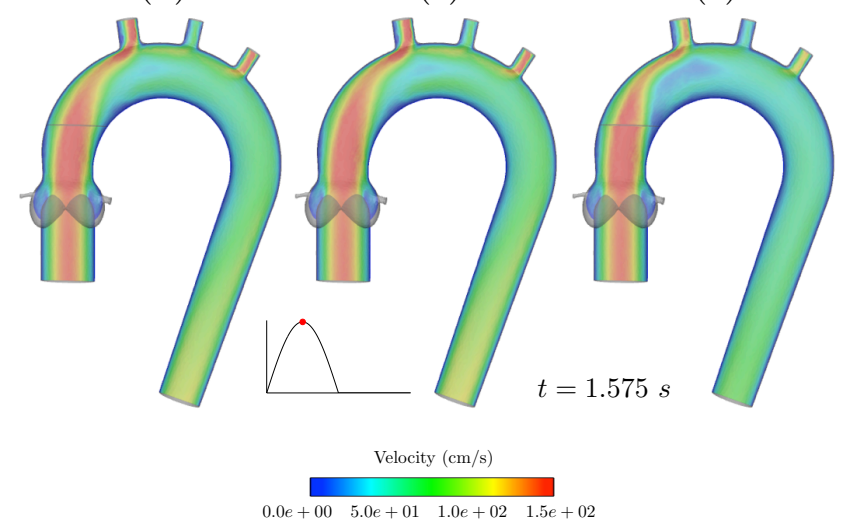

(b)

(c)

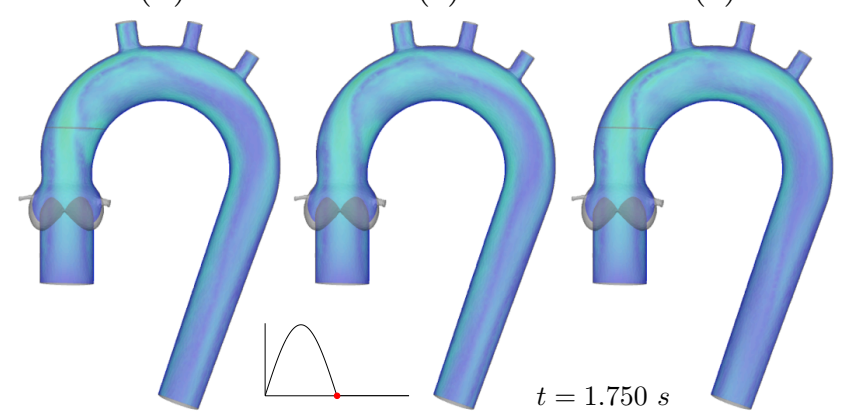

Figure 3: Static pressure formulation. Snapshot of the velocity magnitude at two time instants obtained with: (a) Algorithm 4.2; (b) Reference solution generated from (1); (c) Algorithm 4.3.
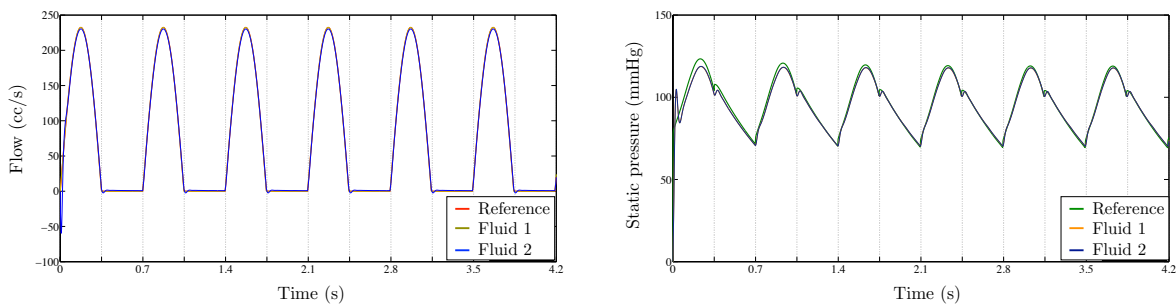

Figure 4: Reference solution generated from (1) vs Algorithm 4.2: interface flows (left) and static pressures (right).

Algorithm 4.3 yields a mass leak on the interface and has very poor results at the outlets. This is due to the non-consistent terms (27) and (28). This behavior is more visible at high velocity, i.e., in the systole phase.

Figures 4 and 5 show the interface flow rate and pressure in $\Omega_{1}$ and $\Omega_{2}$, obtained with Algorithms 4.2 and 4.3, respectively. The results provided by Algorithm 4.2 are clearly more accurate than those obtained with Algorithm 4.3. This can also be inferred from the error indicators reported in Table 2. 

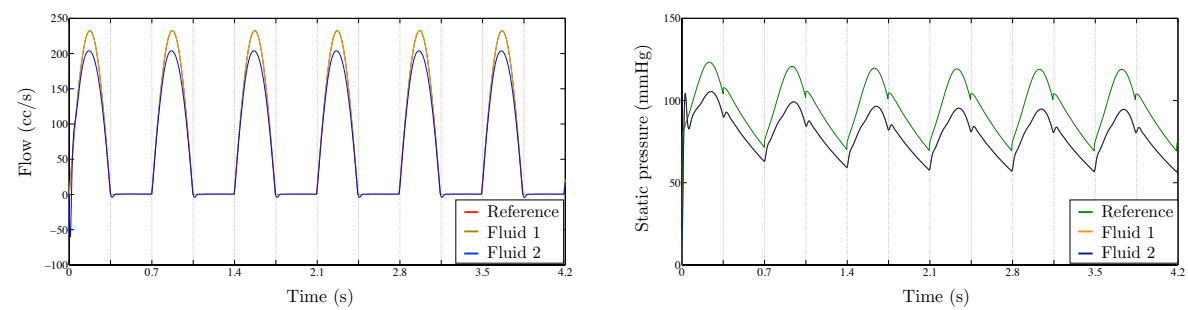

Figure 5: Reference solution generated from (1) vs Algorithm 4.3: interface flows (left) and static pressures (right).

Table 2: Interface relative errors: flow $\varepsilon_{f_{1}}, \varepsilon_{f_{2}}$ and pressure $\varepsilon_{p_{1}}, \varepsilon_{p_{2}}$. Interface drop errors: flow $\varepsilon_{f}$ and pressure $\varepsilon_{p}$.

\begin{tabular}{|c|c||c|c|}
\multicolumn{5}{c|}{ Algorithm 4.2 } \\
\begin{tabular}{|c|c|c|c|}
\hline$\varepsilon_{f_{1}}$ & 0.0048 & $\varepsilon_{p_{1}}$ & 0.0135 \\
\hline$\varepsilon_{f_{2}}$ & 0.0123 & $\varepsilon_{p_{2}}$ & 0.0140 \\
\hline \hline$\varepsilon_{f}$ & 0.0153 & $\varepsilon_{p}$ & 0.0013 \\
\hline
\end{tabular}
\end{tabular}

\begin{tabular}{|c|c||l|l|}
\multicolumn{5}{c}{ Algorithm 4.3 } \\
\begin{tabular}{|c|c|c|c|}
\hline$\varepsilon_{f_{1}}$ & 0.0048 & $\varepsilon_{p_{1}}$ & 0.1864 \\
\hline$\varepsilon_{f_{2}}$ & 0.1058 & $\varepsilon_{p_{2}}$ & 0.1866 \\
\hline \hline$\varepsilon_{f}$ & 0.1054 & $\varepsilon_{p}$ & 0.0012 \\
\hline
\end{tabular}
\end{tabular}
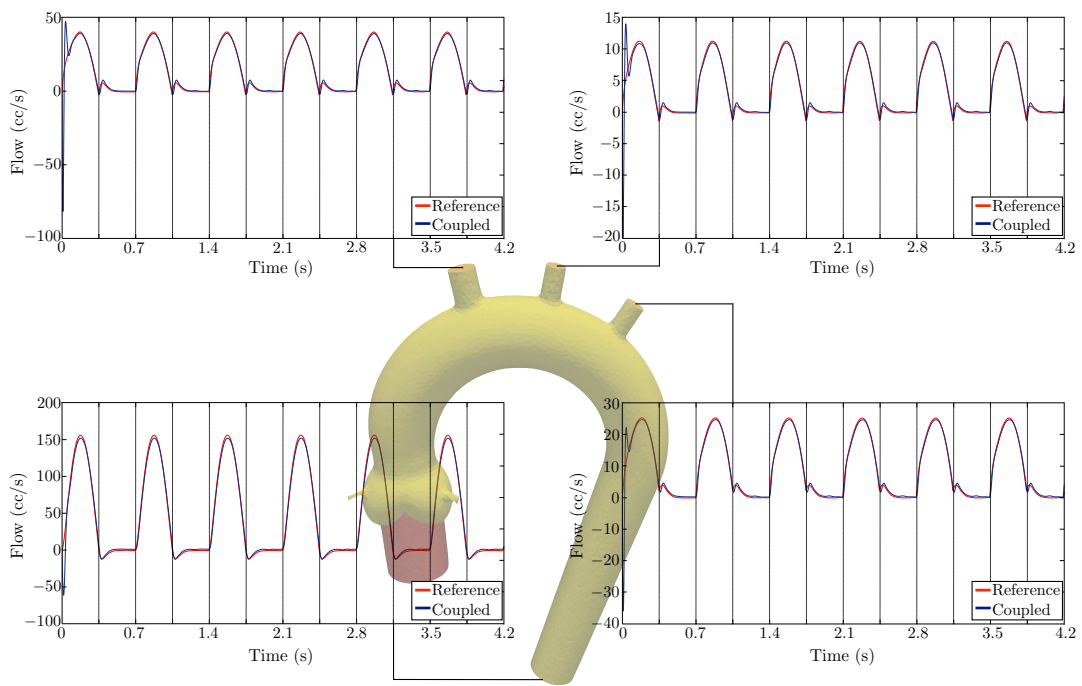

Figure 6: Reference solution generated from (1) vs Algorithm 4.2: outlet flows.

The same behavior is observed for the flow rate and the pressure at the outlets. Figures 6 and 7 and Figures 8 and 9 compare the reference solution with the results obtained with the staggered algorithms in the three top arteries and the descending aorta. As shown by Table 3, the flow rate and pressure errors obtained with Algorithm 4.2 are between $1 \%$ and $5 \%$, whereas with Algorithm 4.3 we get a $20 \%$ error.

\subsubsection{Total pressure formulation}

In this paragraph the results obtained with Algorithm 4.4 are presented. Figure 10 shows snapshots taken at two time instants of the third cardiac cycle, in the maximum of the systole $t=1.575 \mathrm{~s}$ and at the beginning of the diastole $t=1.750 \mathrm{~s}$, for the explicit algorithm with the 

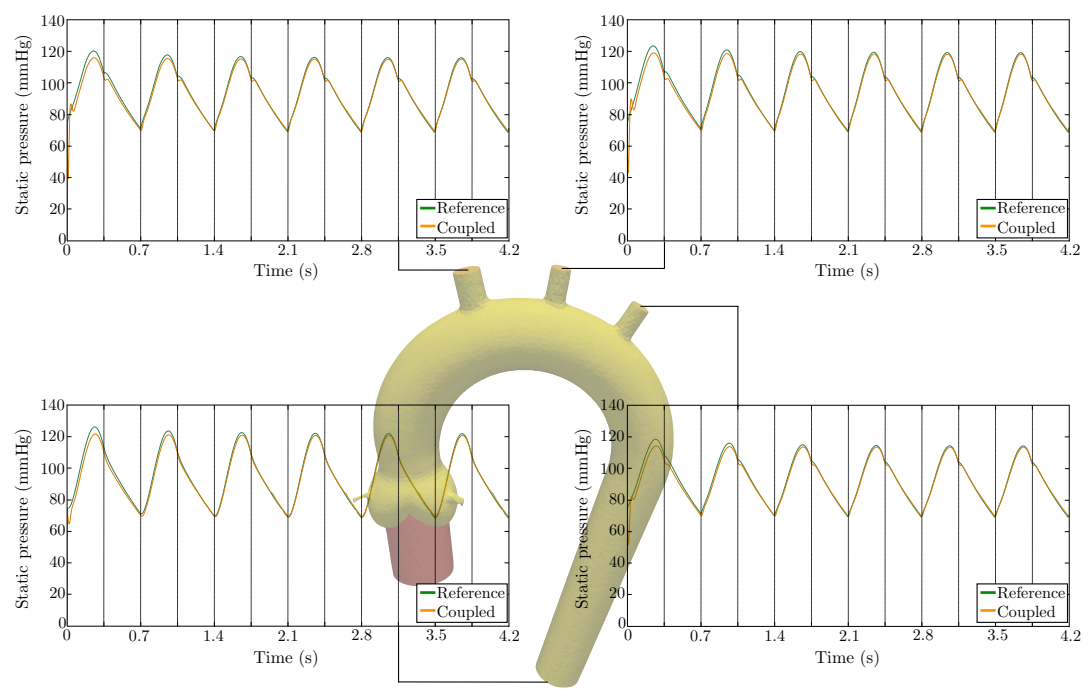

Figure 7: Reference solution generated from (1) vs Algorithm 4.2: outlet static pressures.
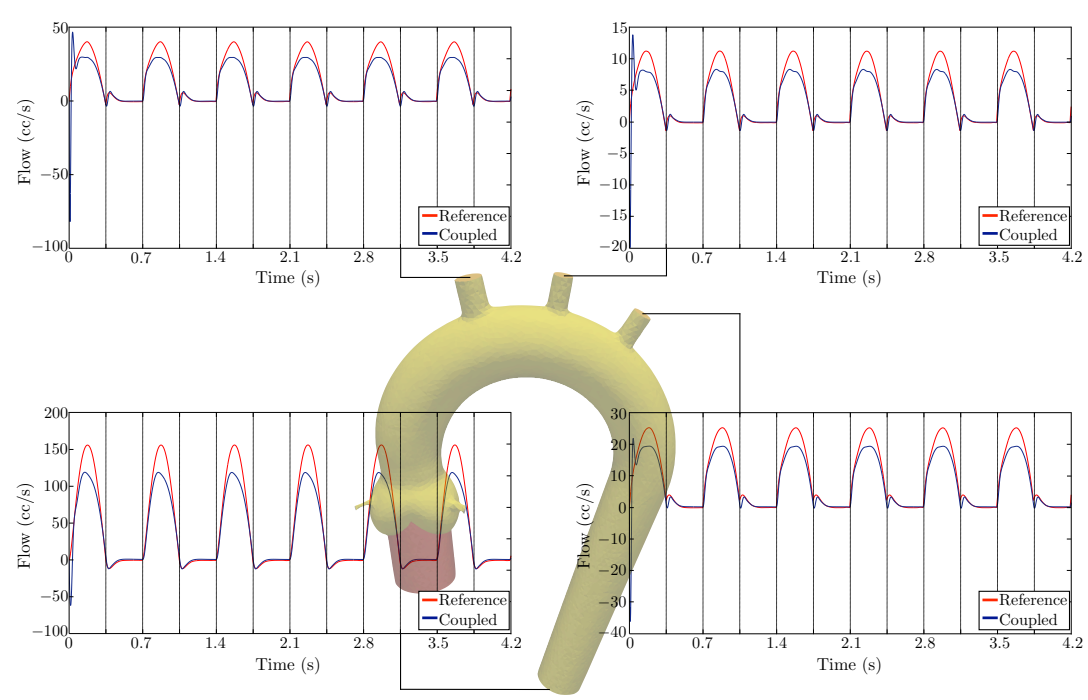

Figure 8: Reference solution generated from (1) vs Algorithm 4.3: outlet flows.

total pressure and the reference solution obtained by the discretization of (31), using a standard conforming finite element method and a semi-implicit monolithic time-stepping. Note the good agreement of the results on the interface and at the outlets.

Figure 11 depicts the flow rate, on the left, and the pressure, on the right, for Fluid 1 and the Fluid 2, compared with the reference solution on the interface. Except very slight differences for Fluid 2 in the peak of the systole and at the beginning of the diastole, the flow rate and the pressure drop between the two fluids are around $2.86 \%$ and $1.13 \%$ respectively (Table 4 ).

The same behavior can be observed for the flow rate and the pressure at the outlets (Figure 12 and 13). Discrepancies still arise at the maximum of the systoles and at the beginning of the 

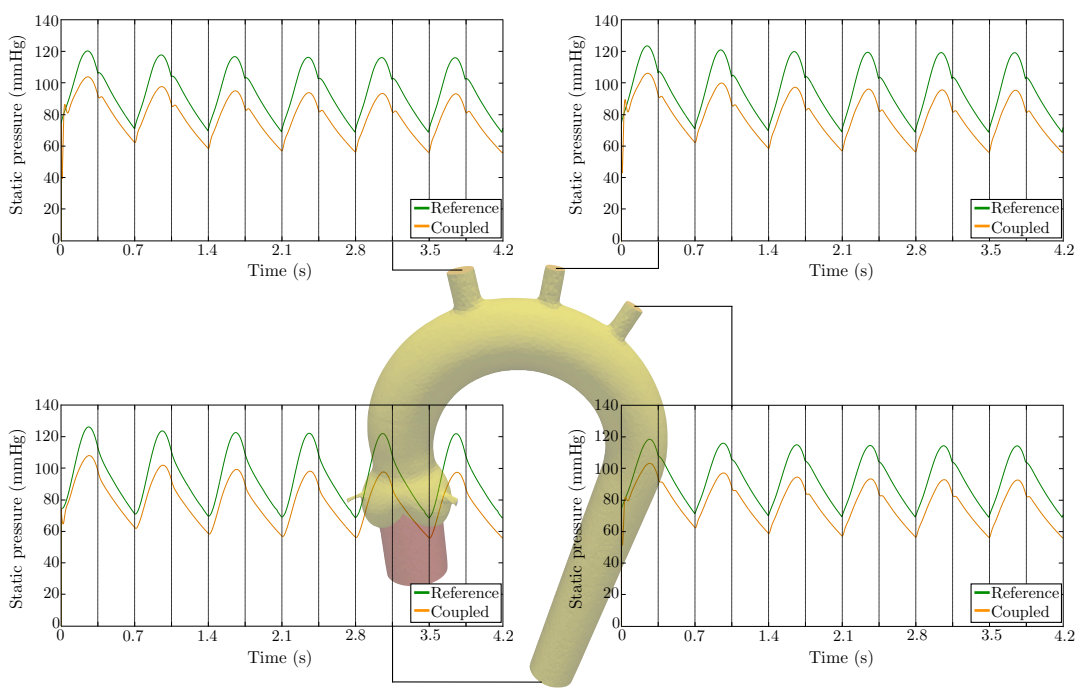

Figure 9: Reference solution generated from (1) vs Algorithm 4.3: outlet static pressures.

Table 3: Outlet flow $\varepsilon_{f_{2}}$ and static pressure $\varepsilon_{p_{2}}$ errors.

\begin{tabular}{|c|c|c|c|c|}
\multicolumn{4}{c}{} & \multicolumn{4}{c|}{ Algorithm 4.2 } \\
\cline { 2 - 5 } \multicolumn{1}{c|}{} & $\Gamma_{a}$ & $\Gamma_{b}$ & $\Gamma_{c}$ & $\Gamma_{\text {out }}$ \\
\hline$\varepsilon_{f_{2}}$ & 0.0589 & 0.0596 & 0.0488 & 0.0411 \\
\hline$\varepsilon_{p_{2}}$ & 0.0115 & 0.0121 & 0.0111 & 0.0122 \\
\hline
\end{tabular}

\begin{tabular}{|c|c|c|c|c|}
\cline { 2 - 5 } \multicolumn{1}{c|}{} & \multicolumn{4}{c}{ Algorithm 4.3} \\
\cline { 2 - 5 } \multicolumn{1}{c|}{} & $\Gamma_{a}$ & $\Gamma_{b}$ & $\Gamma_{c}$ & $\Gamma_{\text {out }}$ \\
\hline$\varepsilon_{f_{2}}$ & 0.2171 & 0.2237 & 0.2003 & 0.2185 \\
\hline$\varepsilon_{p_{2}}$ & 0.1822 & 0.1843 & 0.1797 & 0.1828 \\
\hline
\end{tabular}

(a)

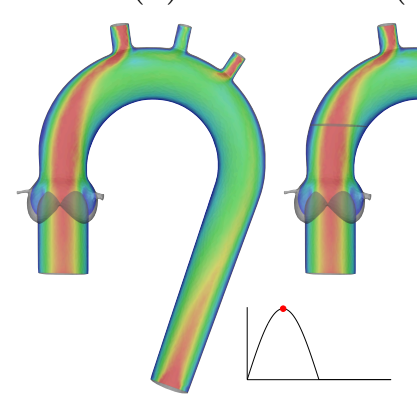

$t=1.575 \mathrm{~s}$ (b)

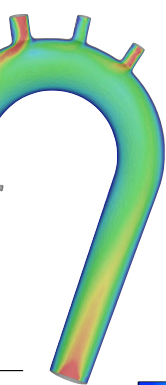

(a)

(b)

Figure 10: Total pressure formulation. Snapshot of the velocity magnitude at two time instants obtained with: Reference solution generated from (31) (a) and Algorithm 4.4 (b).

Table 4: Interface relative errors: flow $\varepsilon_{f_{1}}, \varepsilon_{f_{2}}$ and total pressure $\varepsilon_{\pi_{1}}, \varepsilon_{\pi_{2}}$. Interface drop errors: flow $\varepsilon_{f}$ and total pressure $\varepsilon_{\pi}$.

\begin{tabular}{|c|c||c|c|}
\hline$\varepsilon_{f_{1}}$ & 0.0053 & $\varepsilon_{\pi_{1}}$ & 0.0321 \\
\hline$\varepsilon_{f_{2}}$ & 0.0266 & $\varepsilon_{\pi_{2}}$ & 0.0415 \\
\hline \hline$\varepsilon_{f}$ & 0.0286 & $\varepsilon_{\pi}$ & 0.0113 \\
\hline
\end{tabular}



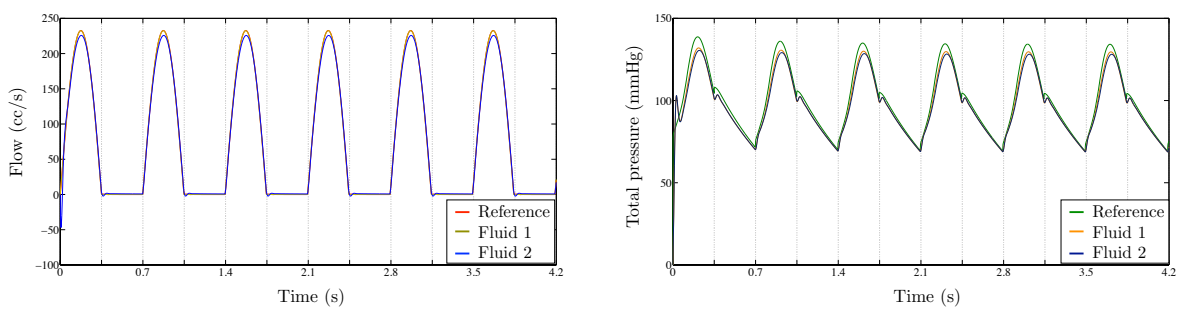

Figure 11: Reference solution generated from (31) vs Algorithm 4.4: interface flows (left) and total pressures (right).
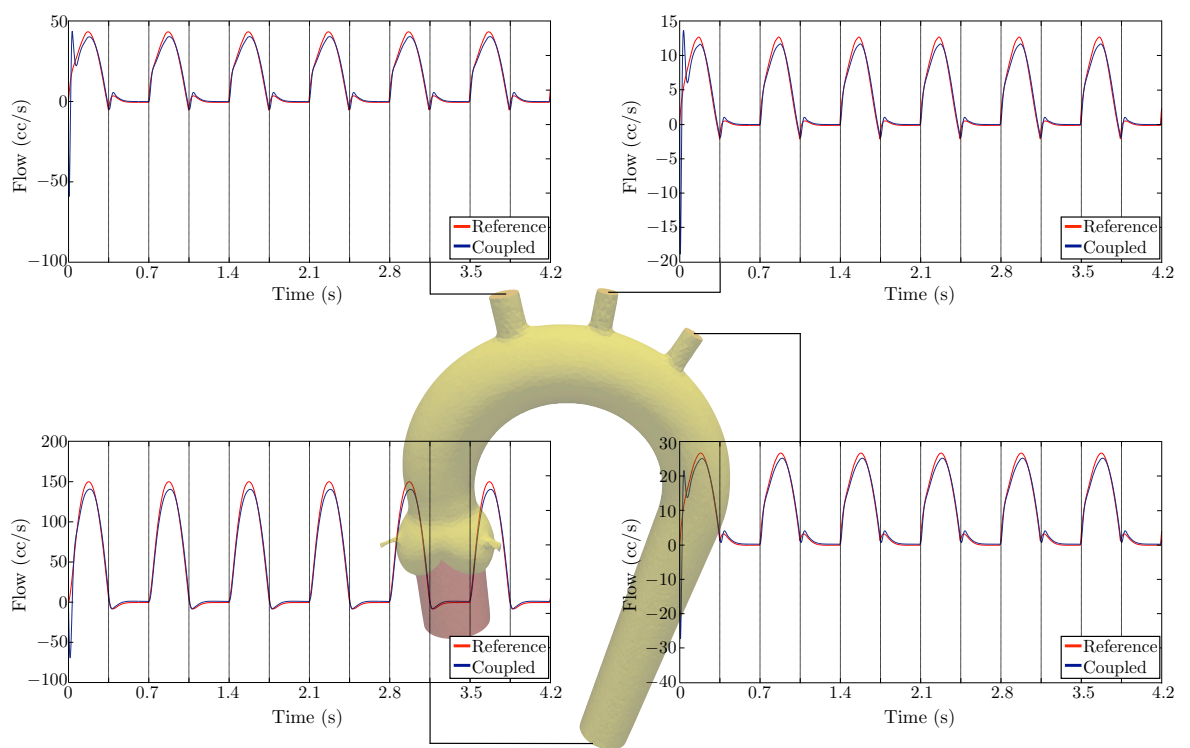

Figure 12: Reference solution generated from (31) vs Algorithm 4.4: outlet flows.

Table 5: Algorithm 4.4: Outlet flow $\varepsilon_{f_{2}}$ and total pressure $\varepsilon_{\pi_{2}}$ errors.

\begin{tabular}{|c|c|c|c|c|}
\cline { 2 - 5 } \multicolumn{1}{c|}{} & $\Gamma_{a}$ & $\Gamma_{b}$ & $\Gamma_{c}$ & $\Gamma_{\text {out }}$ \\
\hline$\varepsilon_{f_{2}}$ & 0.0853 & 0.0926 & 0.0714 & 0.0670 \\
\hline$\varepsilon_{\pi_{2}}$ & 0.0295 & 0.0311 & 0.0276 & 0.0284 \\
\hline
\end{tabular}

diastoles. The errors in these cases remain between $6 \%$ and $9 \%$ for the flow rate and around $3 \%$ for the pressure (Table 5).

In our implementation, and for this specific test case (with an unbalanced number of degrees of freedom in the two sub-domains), the staggered solutions were typically $30 \%$ faster than the monolithic ones.

\subsubsection{Inverted sub-problems and three sub-domains test cases}

As additional tests, the staggered Algorithm 4.2 is tested in the physical configuration described in Section 5.2.1 but with two different repartitions of the sub-domains. The first one is obtained inverting the two sub-problems, i.e the Fluid 1 (20) is solved in the aortic arch and and the 


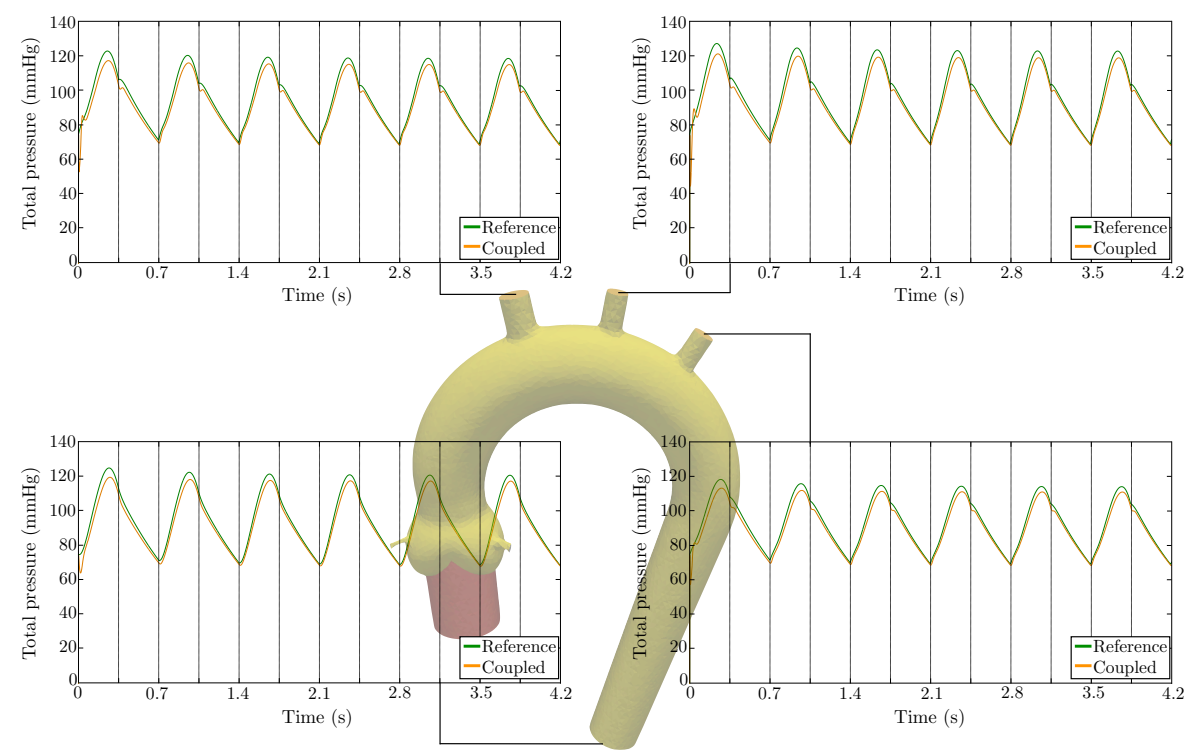

Figure 13: Reference solution generated from (31) vs Algorithm 4.4: outlet total pressures.

Fluid 2 (21) is solved in the aortic root. In the second test case the domain $\Omega$ is split in three non-overlapping sub-domains, the Fluid 1 is solved in the aortic arch, and the Fluid 2 in the aortic root and in the descending aorta. In Figure 14 these two new tests are compared with the two-domain test case of Section 5.2.1. The velocity magnitude is shown at $t=1.575 \mathrm{~s}$ and $t=1.750 \mathrm{~s}$.

The three numerical examples are in very good agreement. This is confirmed if we look at Figure 15 and Figure 16, in which the flow and pressure course in the outlets are compared. The flow and pressure errors in the outlets are reported in Table 6.

Table 6: Outlet flow $\varepsilon_{f_{2}}$ and static pressure $\varepsilon_{p_{2}}$ errors.

\begin{tabular}{|c|c|c|c|c|}
\multicolumn{4}{c}{} & \multicolumn{4}{c}{ Inverted sub-problems } \\
\cline { 2 - 5 } \multicolumn{1}{c|}{$\Gamma_{a}$} & $\Gamma_{b}$ & $\Gamma_{c}$ & $\Gamma_{\text {out }}$ \\
\hline$\varepsilon_{f_{2}}$ & 0.0707 & 0.0729 & 0.0583 & 0.0583 \\
\hline$\varepsilon_{p_{2}}$ & 0.0237 & 0.0248 & 0.0230 & 0.0240 \\
\hline
\end{tabular}

\begin{tabular}{|c|c|c|c|c|}
\cline { 2 - 5 } \multicolumn{1}{c|}{} & $\Gamma_{a}$ & $\Gamma_{b}$ & $\Gamma_{c}$ & $\Gamma_{\text {out }}$ \\
\hline$\varepsilon_{f_{2}}$ & 0.0875 & 0.0940 & 0.0777 & 0.0838 \\
\hline$\varepsilon_{p_{2}}$ & 0.0279 & 0.0317 & 0.0276 & 0.0318 \\
\hline
\end{tabular}

With respect to $\varepsilon_{f_{2}}$ and $\varepsilon_{p_{2}}$ of Algorithm 4.2 presented in Table 3, a slight increasing of the errors can be observed. As expected the error increases with the number of the domain partitions.

\section{Conclusions}

In this work we have discussed several loosely coupled strategies for fluid-fluid interaction problems coupling the incompressible Navier-Stokes equations through standard kinematic/kinetic interface conditions. The main ingredients of the methods considered are:

1. an explicit Robin-Robin treatment of the interface coupling and a suitable weakly consistent artificial compressibility on the interface; 
(a)

(b)

(c)
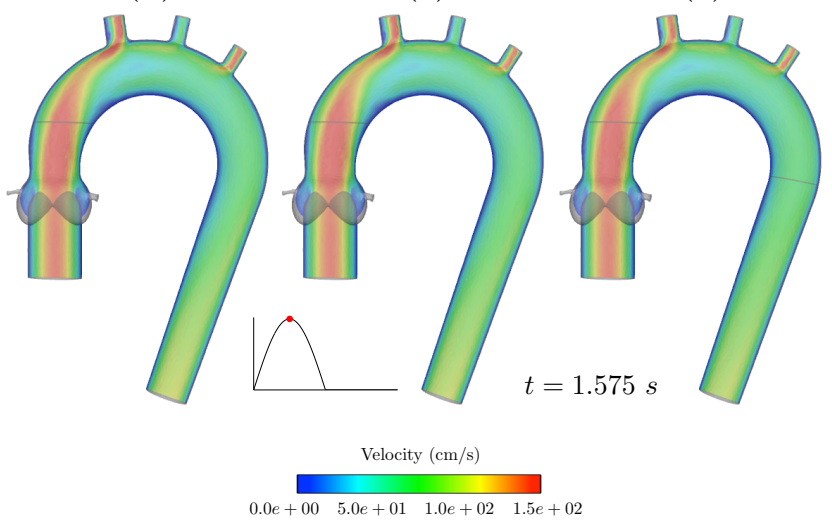

(b)

(c)

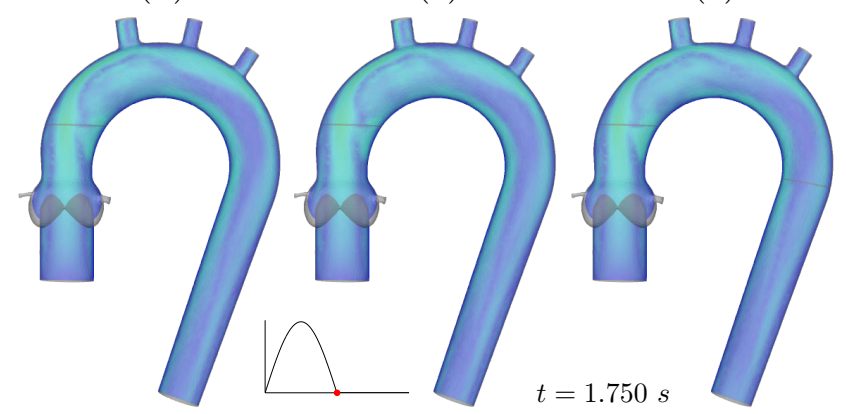

Figure 14: Static pressure formulation. Snapshot of the velocity magnitude at two time instants obtained with Algorithm 4.2 in two non-overlapping sub-domains (a), with inverted sub-problems (b) and in three non-overlapping sub-domains (c).

2. a specific formulation of the convective terms: static pressure (standard and skew-symmetric) and total pressure formulations.

The first controls the artificial power generated by the kinematic/kinetic splitting on the interface, while the second governs the unbalanced dynamic pressure power across the interface.

Energy stability cannot a priori be guaranteed for the standard static pressure formulation Algorithm 4.2. Yet, extensive numerical evidence has shown that the scheme is robust and accurate (with respect to a fully implicit method). This suggests that the artificial dynamic pressure power generated by the splitting has a lower impact on the overall stability of the scheme. Energy stability can be recovered with a static pressure formulation and a skew-symmetric treatment of the convection (Algorithm 4.3). Unfortunately, this formulation is not consistent with the original coupled problem due to its non-confirming character across the interface. The numerical results have confirmed its poor accuracy. For the total pressure formulation, an a priori energy estimate guaranteeing the stability of the splitting (Algorithm 4.4) has been derived. The comparison with fully coupled solutions have shown that the method gives satisfactory results. 

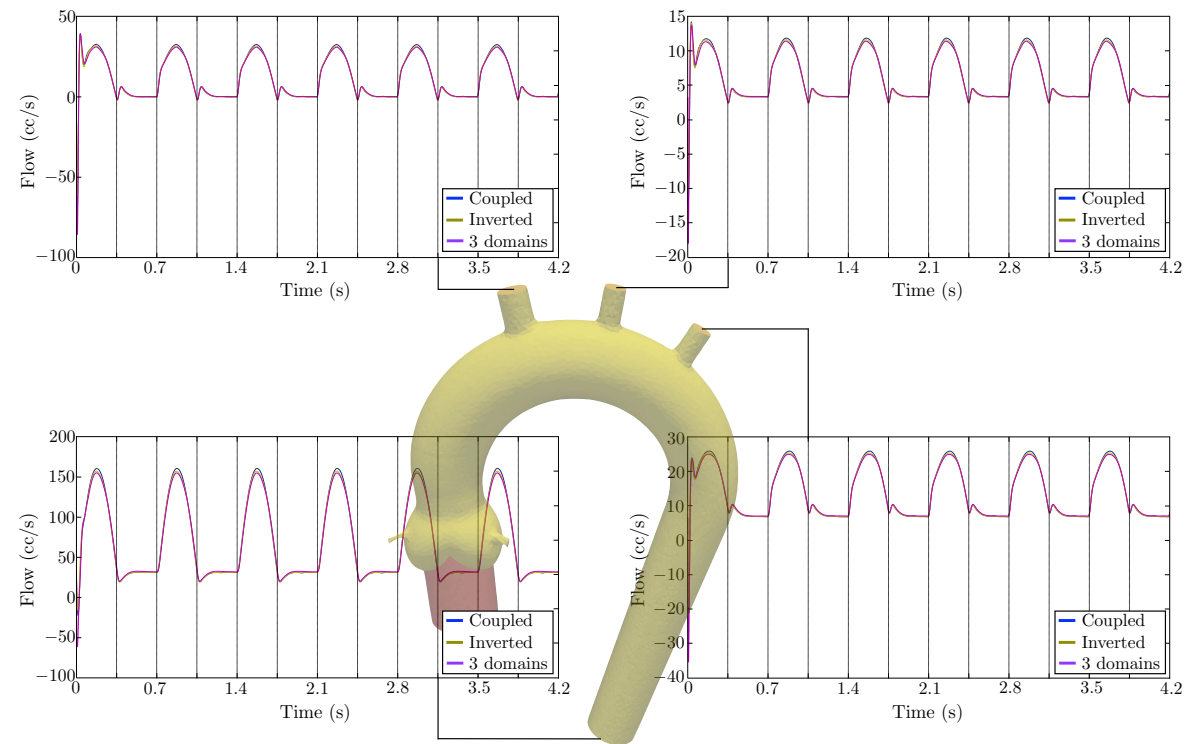

Figure 15: Algorithm 4.2, inverted sub-problems and three sub-domain test cases: outlet flows.
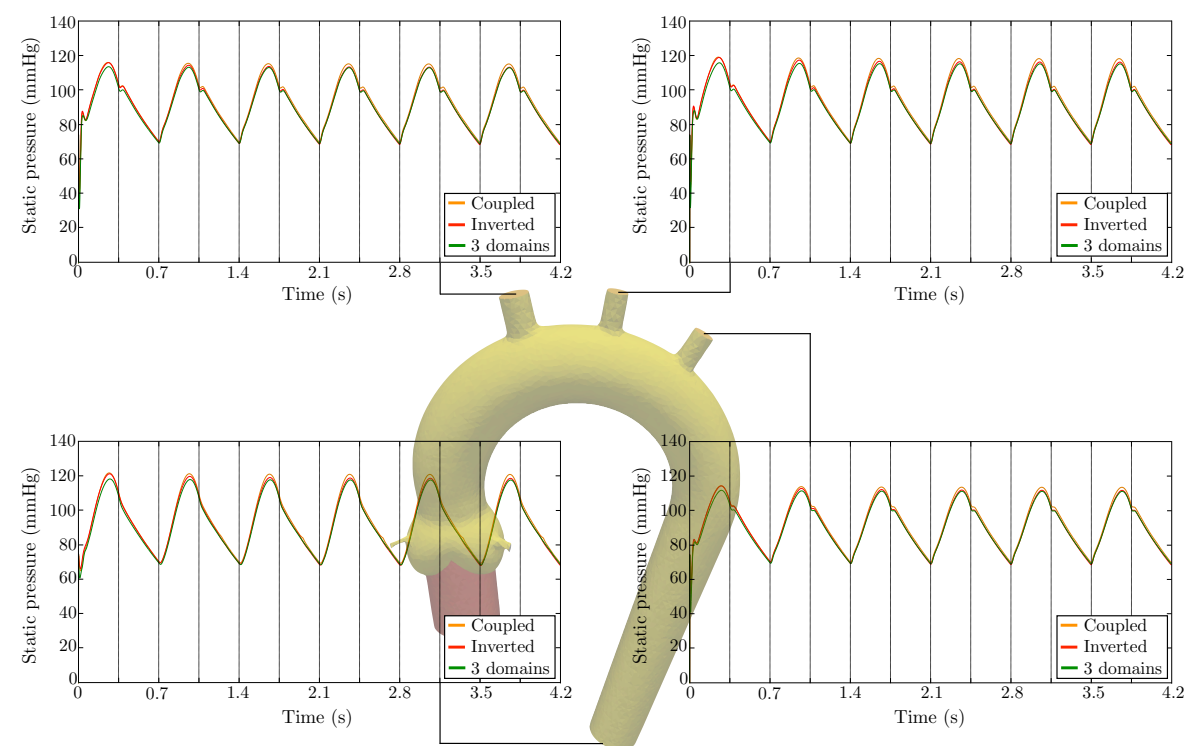

Figure 16: Algorithm 4.2, inverted sub-problems and three sub-domain test cases: outlet static pressures.

\section{References}

[1] M. Astorino, J. Hamers J., S. C., Shadden, and J.-F. Gerbeau. A robust and efficient valve model based on resistive immersed surfaces. Int. J. Numer. Meth. Biomed. Engng., 28:937-959, 2012.

$\mathrm{RR} \mathrm{n}^{\circ} 8415$ 
[2] R. Becker, P. Hansbo, and R. Stenberg. A finite element method for domain decomposition with non-matching grids. M2AN Math. Model. Numer. Anal., 37(2):209-225, 2003.

[3] C. Begue, C. Conca, F. Murat, and O. Pironneau. Les équations des stokes et de NavierStokes avec des conditions limites sur la pression (on Stokes and Navier-stokes equations with boundary condition on the pressure). Non-linear partial differential equations and their applications, Lect. Coll. de France Semin., Paris/Fr. 1985-86, Pitman Res. Notes Math. Ser. 181, IX:179-264, 1988.

[4] C. Bertoglio, A. Caiazzo, and M. A. Fernández. Fractional-step schemes for the coupling of distributed and lumped models in hemodynamics. SIAM Journal on Scientific Computing, $35: 551-575,2013$.

[5] P.J. Blanco, S. Deparis, and A.C.I. Malossi. On the continuity of mean total normal stress in geometrical multiscale cardiovascular problems. Journal of Computational Physics, 251:136155, 2013.

[6] P.J. Blanco, R.A. Feijóo, and S.A. Urquiza. A unified variational approach for coupling 3D1D models and its blood flow applications. Comp. Meth. Appl. Mech. Engrg., 196:4391-4410, 2007.

[7] D. Bresch and J. Koko. Operator-splitting and Lagrange multiplier domain decomposition methods for numerical simulation of two coupled Navier-Stokes fluids. Int. J. Appl. Math. Comput. Sci., 16:419-429, 2006.

[8] E. Burman and M. A. Fernández. Stabilization of explicit coupling in fluid-structure interaction involving fluid incompressibility. Comput. Methods Appl. Mech. Engrg, 198:766-784, 2009 .

[9] E. Burman and M.A. Fernández. An unfitted Nitsche method for incompressible fluidstructure interaction using overlapping meshes. Research Report RR-8424, Inria, 2013. http://hal.inria.fr/hal-00918272.

[10] E. Burman and M.A. Fernández. Explicit strategies for incompressible fluid-structure interaction problems: Nitsche type mortaring versus Robin-Robin coupling. Int. J. Num. Meth. Engrg., 97(10):739-758, 2014.

[11] E. Burman and P. Hansbo. A unified stabilized method for Stokes' and Darcy's equations. J. Comput. Appl. Math., 198:35-51, 2007.

[12] E. Burman and P. Zunino. A domain decomposition method based on interior penalties for advection-diffusion-reaction problems. Siam Jour. Num. Anal., 44:1612-1638, 2006.

[13] J. M. Connors, J. S. Howell, and W. J. Layton. Partitioned time stepping for parabolic two domain problem. SIAM J. Numer Anal., 47:3526-3549, 2009.

[14] J. M. Connors, J. S. Howell, and W. J. Layton. Decoupled time stepping methods for fluid-fluid interaction. SIAM J. Numer Anal., 50(3):1297-1319, 2012.

[15] N. Diniz dos Santos J.-F., Gerbeau, and J.-F. Bourgat. A partitioned fluid-structure algorithm for elastic thin valves with contact. Comput. Methods Appl. Mech. Engrg., 197(1920):1750-1761, 2008. 
[16] L. Formaggia, J.F. Gerbeau, F. Nobile, and A. Quarteroni. On the coupling of 3D and 1D Navier - Stokes equations for flow problems in compliant vessels. Comp. Meth. Appl. Mech. Engrg., 191:561 - 582, 2001.

[17] L. Formaggia, A. Moura, and F. Nobile. On the stability of the coupling of 3D and 1D fluidstructure interaction models for blood flow simulations. ESAIM: Mathematical Modelling and Numerical Analysis, 41(04):743-769, 2007.

[18] L. Grinberg and G. E. Karniadakis. A scalable domain decomposition method for ultraparallel arterial flow simulations. Communications in Computational Physics, 4(5):11511169, 2008.

[19] A. Hansbo, P. Hansbo, and M. G. Larson. A finite element method on composite grids based on Nitsche's method. M2AN Math. Model. Numer. Anal., 37(3):495-514, 2003.

[20] P. Hansbo. Nitsche's method for interface problems in computational mechanics. GAMMMitt., 28(2):183-206, 2005.

[21] P. Hansbo and J. Hermansson. Nitsche's method for coupling non-matching meshes in fluid-structure vibration problems. Comput. Mech., 32(1-2):134-139, 2003.

[22] P. Hansbo, J. Hermansson, and T. Svedberg. Nitsche's method combined with space-time finite elements for ALE fluid-structure interaction problems. Comput. Methods Appl. Mech. Engrg., 193:4195-4206, 2004.

[23] F. Hecht. New development in FreeFem++. J. Numer. Math., 20(3-4):251-265, 2012.

[24] J. G. Heywood, R. Rannacher, and S. Turek. Artificial boundaries and flux and pressure conditions for the incompressible Navier-Stokes equations. International Journal for Numerical Methods in Fluids, 22:325-352, 1996.

[25] W. Layton, C. C. Manica, M. Neda, M. Olshanskii, and L.G. Rebholz. On the accuracy of the rotation form in simulations of the Navier-Stokes equations. Journal of Computational Physics, 228:3433-3447, 2009.

[26] A.C.I. Malossi, P.J. Blanco, S. Deparis, and A. Quarteroni. Algorithms for the partitioned solution of weakly coupled fluid models for cardiovascular flows. International Journal for Numerical Methods in Biomedical Engineering, 27:2035-2057, 2011.

[27] A. Massing, M.G. Larson, A. Logg, and M.E. Rognes. A stabilized Nitsche overlapping mesh method for the Stokes problem. Numer. Math., 2014.

[28] P. Moireau, N. Xiao, M. Astorino, C.A. Figueroa, D. Chapelle, C.A. Taylor, and J.F. Gerbeau. External tissue support and fluid-structure simulation in blood flows. Biomech Model Mechanobiol, 11:1-18, 2012.

[29] J. Nitsche. Über ein Variationsprinzip zur L:̈ung von Dirichlet-Problemen bei Verwendung von Teilräumen, die keinen Randbedingungen unterworfen sind. Abh. Math. Semin. Univ. Hamb., 36:9-15, 1971.

[30] P.Müller. The equation of oceanic motion, volume 2. Cambrige University Press, Cambrige, UK, 1979.

$\mathrm{RR} \mathrm{n}^{\circ} 8415$ 
[31] A. Porpora, P. Zunino, C. Vergara, and M. Piccinelli. Numerical treatment of boundary conditions to replace lateral branches in hemodynamics. International Journal for Numerical Methods in Biomedical Engineering, 28:1165-1183, 2012.

[32] A. Quarteroni and A. Veneziani. Analysis of a geometrical multiscale model based on the coupling of PDE's and ODE's for blood flow simulations. SIAM J Multiscale Model Simul, 1:173 - 195, 2003.

[33] R. Temam. Navier-Stokes Equations, volume 2. Studies in Mathematics and its Applications. North-Holland Publishing Co., Amsterdam, 1979.

[34] V. Thomée. Galerkin Finite Element Methods for Parabolic Problems. Springer Series in Computational Mathematics. Springer-Verlag, Berlin, 2006.

[35] A. Veneziani and C. Vergara. Flow rate defective boundary conditions in haemodynamics simulations. International Journal for Numerical Methods in Fluids, 47(8-9):803-816, 2005. 


\section{Contents}

1 Introduction $\quad 3$

2 Coupled fluid-fluid problem $\quad 4$

3 Space semi-discretization via interface Nitsche's method 4

3.1 Monolithic formulation . . . . . . . . . . . . . . . . . . . 5

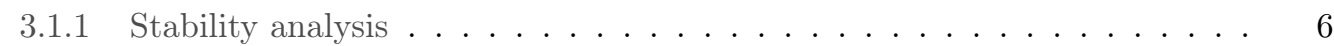

3.2 Partitioned formulation . . . . . . . . . . . . . . . . 8

4 Time-discretization: fully discrete formulations $\quad 8$

4.1 Implicit coupling . . . . . . . . . . . . . . . . . . . . . . . . . . . . . . . . .

4.2 Explicit coupling: a static pressure formulation . . . . . . . . . . . . . . . 10

4.3 Explicit coupling: a stable but inconsistent formulation . . . . . . . . . . . . . . 14

4.4 Explicit coupling: a total pressure formulation . . . . . . . . . . . . . . . 15

5 Numerical Examples $\quad 17$

5.1 Impact of the stabilization parameters . . . . . . . . . . . . . . 18

5.2 Aortic blood flow simulation . . . . . . . . . . . . . . . . . . . 18

5.2 .1 Static pressure formulations . . . . . . . . . . . . . . . . 19

5.2 .2 Total pressure formulation . . . . . . . . . . . . . . . . . 21

5.2.3 Inverted sub-problems and three sub-domains test cases . . . . . . . . . 24

6 Conclusions $\quad 25$ 


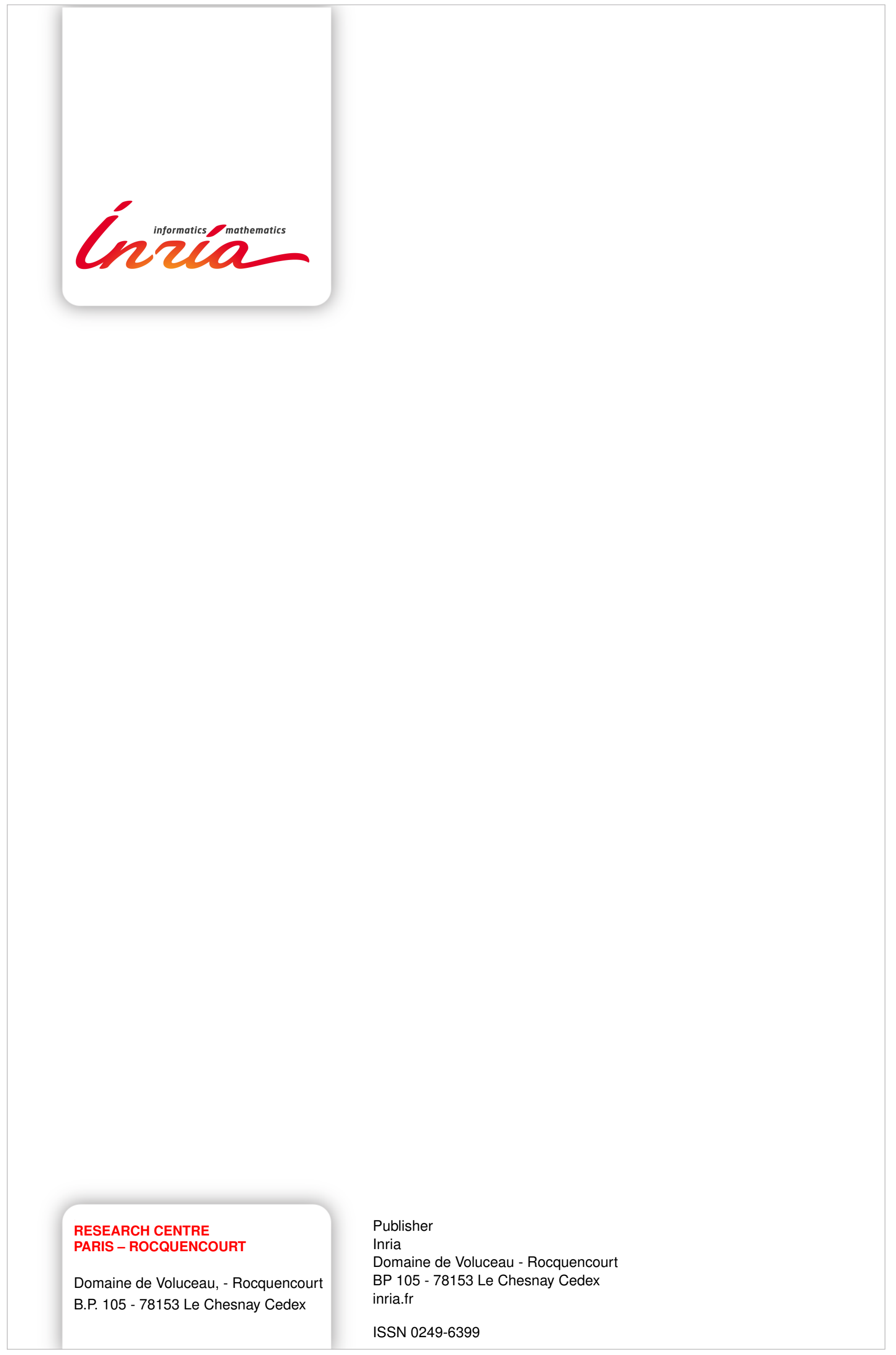

